\title{
Dual Activation in Asymmetric Allylsilane Addition to Chiral $N$-Acylhydrazones: Method Development, Mechanistic Studies, and Elaboration of Homoallylic Amine Adducts
}

\author{
Gregory K. Friestad, ${ }^{\text {a }}$ Chandra Sekhar Korapala, and Hui Ding ${ }^{\mathrm{b}}$ \\ ${ }^{a}$ Department of Chemistry, University of Iowa, Iowa City, Iowa, 52242 \\ ${ }^{b}$ Department of Chemistry, University of Vermont, Burlington, Vermont, 05405 \\ email: gregory-friestad@uiowa.edu
}

\section{Supporting Information}

\section{Table of Contents}

$\begin{array}{ll}\text { Materials and Methods } & \text { S2 }\end{array}$

Preparation and Analysis of Compounds 1, 2, 4, 6-15, 17-26 S2

$\begin{array}{lr}\text { Stereochemical Assignments } & \text { S14 }\end{array}$

Effects of Chiral Ligands on Intramolecular Allylic Amination of $24 . \quad$ S15

$\begin{array}{ll}\text { References } & \text { S16 }\end{array}$

NMR Spectra of 1e, 1f, $\mathbf{2 b}, \mathbf{7}, \mathbf{1 1},(Z)-\mathbf{1 4}, \mathbf{1 5}, \mathbf{1 7}, \mathbf{1 8},(2 S)-\mathbf{2 5}, \mathbf{2 6} \quad$ S17 
Materials and Methods. Reactions employed oven- or flame-dried glassware under nitrogen unless otherwise noted. THF and toluene were distilled from sodium/benzophenone ketyl under argon. $\mathrm{CH}_{2} \mathrm{Cl}_{2}$ was distilled from $\mathrm{CaH}_{2}$ under argon or nitrogen. Nitrogen was passed successively through columns of anhydrous $\mathrm{CaSO}_{4}$ and R3-11 catalyst for removal of water and oxygen, respectively. All other materials were used as received from commercial sources unless otherwise noted. Tetrabutylammonium triphenyldifluorosilicate (TBAT) was prepared according to literature methods, ${ }^{1}$ or commercial TBAT was recrystallized from EtOAc. Thin layer chromatography (TLC) employed glass $0.25 \mathrm{~mm}$ silica gel plates with UV indicator. Flash chromatography columns were packed with 230-400 mesh silica gel as a slurry in the initial elution solvent. Gradient flash chromatography was conducted by adsorption of product mixtures on silica gel, packing over a short pad of clean silica gel as a slurry in hexane, and eluting with a continuous gradient from hexane to the indicated solvent. Radial chromatography refers to centrifugally accelerated thin-layer chromatography performed with a Chromatotron using commercially supplied rotors. Melting points are uncorrected. Nuclear magnetic resonance (NMR) data were obtained at operating frequencies of 500 or $300 \mathrm{MHz}$ for ${ }^{1} \mathrm{H}$ and 125 or $75 \mathrm{MHz}$ for ${ }^{13} \mathrm{C}$. Infrared spectra were recorded using a single beam FT-IR spectrophotometer by standard transmission methods or by use of an attenuated total reflectance (ATR) probe. Optical rotations were determined using a digital polarimeter operating at ambient temperature. Low resolution mass spectra were obtained using sample introduction by dip, liquid chromatography or gas chromatography. High resolution mass spectra and combustion analyses were obtained from external commercial and institutional services. Chromatographic diastereomer ratio analyses employed GCMS with $15 \mathrm{~mL}$ x $0.25 \mathrm{~mm}$ I.D X $0.25 \mu$ F.T 5\%-phenyl-95\%-dimethylsiloxane column and helium as mobile phase or HPLC with Microsorb-MV Si 8um 100A or Chiralcel OD columns (2-propanol/hexane as mobile phase) or Chirex 3014 column (chloroform/hexane as mobile phase).

Note regarding specific optical rotations: Several specific rotations $[\alpha]_{D}$ of compounds $\mathbf{1 b}-\mathbf{1 f}$, 2a-2f, and 4 were recorded erroneously in the supporting information of the preliminary report. ${ }^{2}$ Corrected $[\alpha]_{D}$ data are reported herein.

Preparation of enantiopure $\boldsymbol{N}$-acylhydrazones (General Procedure A): A procedure based on the literature method $^{3}$ was employed. To a solution of $(S)$-3-amino-4-phenylmethyl-2-oxazolidinone $(0.1-0.3 \mathrm{mmol})$ in toluene $(10 \mathrm{~mL})$ was added $\mathrm{MgSO}_{4}(200 \mathrm{mg})$, a catalytic amount of $p$-TsOH (ca. 5 mol\%) and aldehyde (ca. 1.5 equiv). After heating at reflux for $10 \mathrm{~min}$, concentration and flash chromatography (5:1 hexane/ethyl acetate) gave pure hydrazones $\mathbf{1 a - 1 h}$.

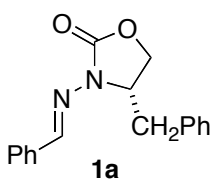

(S)-3-(Benzylidene)amino-4-phenylmethyl-2-oxazolidinone (1a). From $(S)$-3amino-4-phenylmethyl-2-oxazolidinone $(60 \mathrm{mg}, 0.31 \mathrm{mmol})$ and benzaldehyde $(0.05 \mathrm{~mL}$, $0.49 \mathrm{mmol}$ ) by General Procedure A was obtained 1a (70 $\mathrm{mg}, 81 \%)$ as a colorless solid, the properties of which matched the published data. ${ }^{4}$

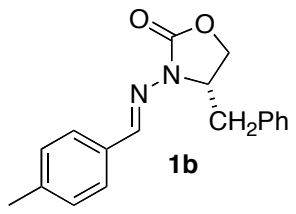

(S)-3-(p-Methylbenzylidene)-amino-4-phenylmethyl-2-oxazolidinone (1b). From $(S)$-3-amino-4-phenylmethyl-2-oxazolidinone (43 $\mathrm{mg}, 0.22 \mathrm{mmol}$ ) and $p$ tolualdehyde $(0.04 \mathrm{~mL}, 0.34 \mathrm{mmol})$ by General Procedure A was obtained $\mathbf{1 b}(62$ $\mathrm{mg}, 94 \%)$ as a colorless solid; mp $111-112^{\circ} \mathrm{C} ;[\alpha]_{\mathrm{D}}{ }^{20}-22^{\circ}\left(c 0.11, \mathrm{CHCl}_{3}\right) ; \mathrm{IR}$ (film) $3027,2918,1760,1615,1497,1474,1455,1403,1212,1089 \mathrm{~cm}^{-1} ;{ }^{1} \mathrm{H}$ NMR (500 $\left.\mathrm{MHz}, \mathrm{CDCl}_{3}\right) \delta 8.87(\mathrm{~s}, 1 \mathrm{H}), 7.63-7.04(\mathrm{~m}, 9 \mathrm{H}), 4.50-4.43(\mathrm{~m}, 1 \mathrm{H}), 4.28(\mathrm{dd}, J=8.0,8.0 \mathrm{~Hz}, 1 \mathrm{H}), 4.12$ $(\mathrm{dd}, J=8.8,5.6 \mathrm{~Hz}, 1 \mathrm{H}), 3.30(\mathrm{dd}, J=13.9,3.6 \mathrm{~Hz}, 1 \mathrm{H}), 2.90(\mathrm{dd}, J=13.9,8.6 \mathrm{~Hz}, 1 \mathrm{H}), 2.43(\mathrm{~s}, 3 \mathrm{H})$; ${ }^{13} \mathrm{C} \mathrm{NMR}\left(125 \mathrm{MHz} \mathrm{CDCl}_{3}\right) \delta 153.9,151.2,140.8,135.5,131.8,129.5,129.4,128.9,127.5,127.3$, 
65.8, 58.4, 37.8, 21.5; MS (CI) $m / z$ (relative intensity) $295\left([\mathrm{M}+\mathrm{H}]^{+}, 100 \%\right)$; Anal. Calcd. for $\mathrm{C}_{18} \mathrm{H}_{18} \mathrm{~N}_{2} \mathrm{O}_{2}$ : C, 73.45; H, 6.16; N, 9.52. Found: C, 73.31; H, 6.25; N, 9.25.

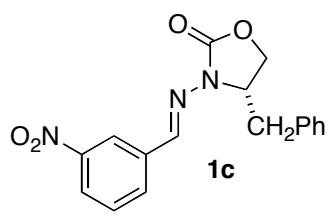

(S)-3-(3'-Nitrobenzylidene)amino-4-phenylmethyl-2-oxazolidinone (1c). From $(S)$-3-amino-4-phenylmethyl-2-oxazolidinone $(50 \mathrm{mg}, 0.26 \mathrm{mmol})$ and $\mathrm{m}$ nitrobenzaldehyde $(0.06 \mathrm{~mL}, 0.40 \mathrm{mmol})$ by General Procedure A was obtained $\mathbf{1 f}$ (75 mg, 91\%) as a colorless solid; $\mathrm{mp} 148-150{ }^{\circ} \mathrm{C} ;[\alpha]_{\mathrm{D}}{ }^{23}-5.5^{\circ}\left(c 0.29, \mathrm{CHCl}_{3}\right)$; IR (film) 3057, 2921, 1754, 1534, 1399, 1352, 1266, $1099 \mathrm{~cm}^{-1} ;{ }^{1} \mathrm{H}$ NMR $(500 \mathrm{MHz}$, $\left.\mathrm{CDCl}_{3}\right) \delta 9.07(\mathrm{~s}, 1 \mathrm{H}), 8.51(\mathrm{~s}, 1 \mathrm{H}), 8.21$ (dddd, $\left.J=8.2,1.0,1.0,1.0 \mathrm{~Hz}, 1 \mathrm{H}\right), 7.98(\mathrm{dd}, J=7.7,0.9 \mathrm{~Hz}$, $1 \mathrm{H}), 7.57(\mathrm{dd}, J=7.7,7.7 \mathrm{~Hz}, 1 \mathrm{H}), 7.34-7.16(\mathrm{~m}, 5 \mathrm{H}), 4.58-4.53(\mathrm{~m}, 1 \mathrm{H}), 4.36(\mathrm{dd}, J=8.1,8.1 \mathrm{~Hz}, 1 \mathrm{H})$, $4.17(\mathrm{dd}, J=8.2,5.6 \mathrm{~Hz}, 1 \mathrm{H}), 3.32(\mathrm{dd}, J=13.9,4.1 \mathrm{~Hz}, 1 \mathrm{H}), 2.95(\mathrm{dd}, J=13.9,8.4 \mathrm{~Hz}, 1 \mathrm{H}) ;{ }^{13} \mathrm{C} \mathrm{NMR}$ $\left(125 \mathrm{MHz}, \mathrm{CDCl}_{3}\right) \delta 153.5,148.8,136.5,135.1,133.0,129.7,129.4,129.0,127.4,124.6,121.7,66.0$, 58.6, 38.2; MS (EI) $\mathrm{m} / z$ (relative intensity) $325\left(\mathrm{M}^{+}, 29 \%\right.$ ); Anal. Calcd. for $\mathrm{C}_{17} \mathrm{H}_{15} \mathrm{~N}_{3} \mathrm{O}_{4}$ : C, 62.76; $\mathrm{H}$, 4.65; N, 12.92. Found: C, 62.64; H, 4.77; N, 12.72 .

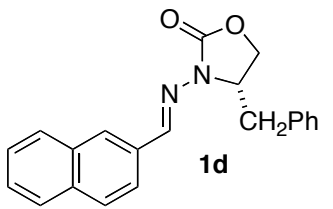

(S)-3-(2'-Naphthylidene)-amino-4-phenylmethyl-2-oxazolidinone (1d). From $(S)$-3-amino-4-phenylmethyl-2-oxazolidinone (43 $\mathrm{mg}, 0.22 \mathrm{mmol}$ ) and 2naphthaldehyde $(52 \mathrm{mg}, 0.33 \mathrm{mmol}$ ) by General Procedure A was obtained 1d (67 $\mathrm{mg}, 92 \%)$ as a colorless solid; $\mathrm{mp} 147-148^{\circ} \mathrm{C} ;[\alpha]_{\mathrm{D}}{ }^{20}-38^{\circ}\left(c 0.12, \mathrm{CHCl}_{3}\right)$; IR (film) 3027, 2918, 2850, 1752, 1651, 1456, 1397, 1265, 1222, $1093 \mathrm{~cm}^{-1} ;{ }^{1} \mathrm{H} \mathrm{NMR}$ $\left(500 \mathrm{MHz}, \mathrm{CDCl}_{3}\right) \delta 9.06(\mathrm{~s}, 1 \mathrm{H}), 8.02-7.17(\mathrm{~m}, 12 \mathrm{H}), 4.53-4.45(\mathrm{~m}, 1 \mathrm{H}), 4.35(\mathrm{dd}, J=8.7,8.7 \mathrm{~Hz}, 1 \mathrm{H})$, $4.19(\mathrm{dd}, J=8.8,5.5 \mathrm{~Hz}, 1 \mathrm{H}), 3.38(\mathrm{dd}, J=13.9,3.8 \mathrm{~Hz}, 1 \mathrm{H}), 2.92(\mathrm{dd}, J=13.9,8.7 \mathrm{~Hz}, 1 \mathrm{H}) ;{ }^{13} \mathrm{C}$ NMR $\left(125 \mathrm{MHz}_{1} \mathrm{CDCl}_{3}\right) \delta 153.9,150.5,135.4,134.5,133.2,132.1,129.6,129.4,129.0,127.5,128.6,127.9$, 127.3, 127.2, 126.7, 122.8, 65.8, 58.2, 37.7; MS (CI) $\mathrm{m} / z$ (relative intensity) $330\left(\mathrm{M}^{+}, 25 \%\right.$ ); Anal. Calcd. for $\mathrm{C}_{21} \mathrm{H}_{18} \mathrm{~N}_{2} \mathrm{O}_{2}$ : C, 76.34; H, 5.49; N, 8.48. Found: C, 76.63; H, 5.50; N, 8.47.

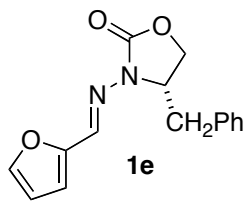

(S)-3-(2'-Furanylidene)amino-4-phenylmethyl-2-oxazolidinone (1e). From $(S)$ 3-amino-4-phenylmethyl-2-oxazolidinone (48 $\mathrm{mg}, 0.25 \mathrm{mmol})$ and 2-furaldehyde (0.06 $\mathrm{mL}, 0.41 \mathrm{mmol})$ by General Procedure A was obtained 1e $(62 \mathrm{mg}, 92 \%)$ as an air- and light-sensitive pale yellow oil; $[\alpha]_{\mathrm{D}}{ }^{20}-65^{\circ}\left(c 0.06, \mathrm{CHCl}_{3}\right)$; IR (film) 3028, 2917, 2849, 1751, 1715, 1456, 1399, 1266, 1224, $1095 \mathrm{~cm}^{-1} ;{ }^{1} \mathrm{H}$ NMR $\left(500 \mathrm{MHz}, \mathrm{CDCl}_{3}\right) \delta 8.96(\mathrm{~s}$, $1 \mathrm{H}), 7.55(\mathrm{~d}, J=1.7 \mathrm{~Hz}, 1 \mathrm{H}), 7.34-7.19(\mathrm{~m}, 5 \mathrm{H}), 6.78(\mathrm{dd}, J=3.4,0.9 \mathrm{~Hz}, 1 \mathrm{H}), 6.56(\mathrm{dd}, J=3.4,1.7$ $\mathrm{Hz}, 1 \mathrm{H}), 4.49-4.45(\mathrm{~m}, 1 \mathrm{H}), 4.29(\mathrm{dd}, J=8.2,8.2 \mathrm{~Hz}, 1 \mathrm{H}), 4.12(\mathrm{dd}, J=8.9,5.9 \mathrm{~Hz}, 1 \mathrm{H}), 3.35(\mathrm{dd}, J=$ 13.9, $3.7 \mathrm{~Hz}, 1 \mathrm{H}), 2.92$ (dd, J = 13.9, $8.9 \mathrm{~Hz}, 1 \mathrm{H}) ;{ }^{13} \mathrm{C}$ NMR $\left(125 \mathrm{MHz}, \mathrm{CDCl}_{3}\right) \delta 153.8,149.7,144.8$, 141.3, 135.2, 129.4, 128.9, 127.3, 114.3, 111.9, 65.7, 58.8, 37.9; MS (CI) $\mathrm{m} / z$ (relative intensity) 295 $\left([\mathrm{M}+\mathrm{H}]^{+}, 100 \%\right)$; HRMS (FAB) $m / z:[\mathrm{M}+\mathrm{H}]^{+}$calcd. for $\mathrm{C}_{15} \mathrm{H}_{15} \mathrm{~N}_{2} \mathrm{O}_{3}$ 271.1083; found, 271.1066.

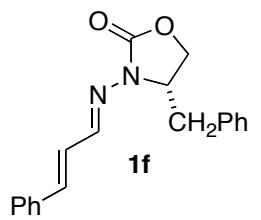

(S)-3-(trans-Cinnamylidene)amino-4-phenylmethyl-2-oxazolidinone (1f). From $(S)$-3-amino-4-phenylmethyl-2-oxazolidinone $(48 \mathrm{mg}, 0.25 \mathrm{mmol})$ and transcinnamaldehyde $(0.05 \mathrm{~mL}, 0.40 \mathrm{mmol})$ by General Procedure A was obtained $\mathbf{1 f}(74 \mathrm{mg}$, $97 \%$ ) as a colorless solid; mp 88-90 ${ }^{\circ} \mathrm{C}$; $[\alpha]_{\mathrm{D}}{ }^{20}-71^{\circ}\left(c 0.08, \mathrm{CHCl}_{3}\right)$; IR (film) 3055, 2919, 2850, 1751, 1713, 1627, 1451, 1400, 1266, 1214, $1091 \mathrm{~cm}^{-1} ;{ }^{1} \mathrm{H}$ NMR $(500 \mathrm{MHz}$, $\left.\mathrm{CDCl}_{3}\right) \delta 8.55(\mathrm{dd}, J=7.9,7.9 \mathrm{~Hz}, 1 \mathrm{H}), 7.50(\mathrm{dd}, J=1.5,1.5 \mathrm{~Hz}, 2 \mathrm{H}), 7.47-6.96(\mathrm{~m}, 10 \mathrm{H}), 4.47-4.42$ $(\mathrm{m}, 1 \mathrm{H}), 4.27(\mathrm{dd}, J=8.8,8.8 \mathrm{~Hz}, 1 \mathrm{H}), 4.14(\mathrm{dd}, J=8.8,5.1 \mathrm{~Hz}, 1 \mathrm{H}), 3.26(\mathrm{dd}, J=13.9,3.3 \mathrm{~Hz}, 1 \mathrm{H})$, $2.92(\mathrm{dd}, J=13.9,8.8 \mathrm{~Hz}, 1 \mathrm{H}) ;{ }^{13} \mathrm{C} \mathrm{NMR}\left(125 \mathrm{MHz}, \mathrm{CDCl}_{3}\right) \delta 153.9,151.2,140.8,135.6,131.8,129.5$, 129.4, 128.9, 127.5, 127.3, 65.8, 58.4, 37.8; MS (CI) $\mathrm{m} / \mathrm{z}$ (relative intensity) $306\left(\mathrm{M}^{+}, 25 \%\right)$. 
Friestad et al.

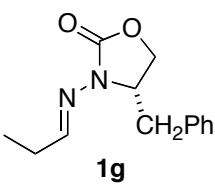

(S)-3-(Propylidene)amino-4-phenylmethyl-2-oxazolidinone (1g). From $(S)$-3amino-4-phenylmethyl-2-oxazolidinone $(27 \mathrm{mg}, 0.14 \mathrm{mmol})$ and propionaldehyde $(0.02$ $\mathrm{mL}, 0.27 \mathrm{mmol}$ ) by General Procedure A was obtained $1 \mathrm{~g}(30 \mathrm{mg}, 92 \%)$ as colorless oil. $^{4}$

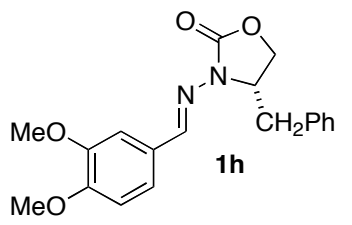

(S)-3-(3,4-Dimethoxybenzylidene)amino-4-phenylmethyl-2-oxazolidinone (1h). From (S)-3-amino-4-phenylmethyl-2-oxazolidinone $(600 \mathrm{mg}, 3.1 \mathrm{mmol})$ and veratraldehyde $(570 \mathrm{mg}, 3.4 \mathrm{mmol})$ by General Procedure A was obtained $\mathbf{1 h}(1.0$ g, 98\%) as a colorless solid; $\mathrm{mp} 113-115{ }^{\circ} \mathrm{C} ;[\alpha]_{\mathrm{D}}^{23}-56.1^{\circ}\left(c 0.18, \mathrm{CHCl}_{3}\right)$; IR (film) 2922, 2851, 1764, 1601, 1514, 1457, 1403, 1267, 1218, 1139, 1089, 1023 $\mathrm{cm}^{-1} ;{ }^{1} \mathrm{H}$ NMR $\left(500 \mathrm{MHz}, \mathrm{CDCl}_{3}\right)$ d $8.75(\mathrm{~s}, 1 \mathrm{H}), 7.34-7.13(\mathrm{~m}, 7 \mathrm{H}), 6.86(\mathrm{~d}, J=8.0 \mathrm{~Hz}, 1 \mathrm{H}), 4.48-4.45$ $(\mathrm{m}, 1 \mathrm{H}), 4.28(\mathrm{dd}, J=8.5,8.5 \mathrm{~Hz}, 1 \mathrm{H}), 4.11(\mathrm{dd}, J=8.8,5.6 \mathrm{~Hz}, 1 \mathrm{H}), 3.90(\mathrm{~s}, 3 \mathrm{H}), 3.88(\mathrm{~s}, 3 \mathrm{H}), 3.28$ (dd, $J=14.0,4.0 \mathrm{~Hz}, 1 \mathrm{H}), 2.90(\mathrm{dd}, J=13.5,8.5 \mathrm{~Hz}, 1 \mathrm{H}) ;{ }^{13} \mathrm{C} \mathrm{NMR}\left(125 \mathrm{MHz}, \mathrm{CDCl}_{3}\right) \delta 154.0,151.4$, $149.5,135.5,129.4,128.9,127.4,127.2,122.5,110.8,108.5,65.8,58.4,56.0$ (2C), 37.8; MS (EI) $\mathrm{m} / \mathrm{z}$ (relative intensity) $325\left(\mathrm{M}^{+}, 29 \%\right)$; Anal. Calcd. for $\mathrm{C}_{19} \mathrm{H}_{20} \mathrm{~N}_{2} \mathrm{O}_{4}$ : C, 67.05; H, 5.92; N, 8.23. Found: C, $66.90 ; \mathrm{H}, 5.86 ; \mathrm{N}, 8.12$.

\section{Allyl Addition to Hydrazones: General Procedures B-D}

Addition using allyltrimethylsilane and TBAF (General Procedure B): To a mixture of hydrazone $(0.3 \mathrm{mmol})$, allyltrimethylsilane $(0.36 \mathrm{mmol})$ and $4 \AA$ molecular sieves in THF $(2 \mathrm{~mL})$ was added TBAF ( $30 \mu \mathrm{L}, 1.0 \mathrm{M}$ in THF, $0.03 \mathrm{mmol})$. The reaction mixture was stirred for $20 \mathrm{~h}$, and filtered on a short silica gel column. The filtrate was concentrated and chromatographed (4:1 hexane/ethyl acetate) to give pure hydrazines $\mathbf{2 a}$.

Addition using allylmagnesium bromide (General Procedure C): A mixture of hydrazone ( 0.2 mmol) and indium triflate $(0.26 \mathrm{mmol})$ in $\mathrm{CH}_{2} \mathrm{Cl}_{2}(2.2 \mathrm{~mL})$ was stirred at room temperature for $2 \mathrm{~h}$. Then, allylmagnesium bromide $\left(0.6 \mathrm{~mL}, 1.0 \mathrm{M}_{\text {in }} \mathrm{Et}_{2} \mathrm{O}, 0.6 \mathrm{mmol}\right)$ was added dropwise. After $2 \mathrm{~d}$, the reaction was quenched by $0.5 \% \mathrm{HCl}$ solution. The organic phase was separated, dried over $\mathrm{MgSO}_{4}$, and purified by flash chromatography to give pure hydrazines 2 .

Addition using tetraallylsilane and TBAT (General Procedure D): A mixture of hydrazone $(0.2$ $\mathrm{mmol})$ and indium triflate $(0.26 \mathrm{mmol})$ in $\mathrm{CH}_{2} \mathrm{Cl}_{2}(2.2 \mathrm{~mL})$ was stirred at room temperature. Meanwhile, a mixture of TBAT $(0.6 \mathrm{mmol})$ and tetraallylsilane $(0.6 \mathrm{mmol})$ in $\mathrm{CH}_{2} \mathrm{Cl}_{2}(0.8 \mathrm{~mL})$ was stirred in another flask. After $4 \mathrm{~h}$, the TBAT mixture was added to the hydrazone mixture. After $2 \mathrm{~d}, 2 \mathrm{~mL}$ of water was added. The organic phase was separated, dried over $\mathrm{MgSO}_{4}$, concentrated and purified by flash chromatography to give pure hydrazines 2a. Diastereomeric ratios were determined by HPLC (MICROSORB-MV ${ }^{\mathrm{TM}} \mathrm{C} 8$ column, eluted with 95:5 hexane/isopropanol) in comparison with authentic mixtures (dr ca. 5:1) obtained in less selective addition reactions (i.e., by General Procedure C). One example is given below for the minor diastereomer of $\mathbf{2 a}$; other minor diastereomers were not separated in quantities sufficient for full characterization.

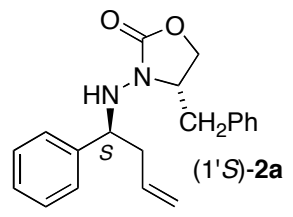

(4S,1'S)-3-(1'-Allyl-1'-phenylmethyl-amino)-4-phenylmethyl-2-oxazolidinone (2a). From 1a (56 mg, $0.2 \mathrm{mmol})$ by General Procedure D was obtained 2a $(50 \mathrm{mg}$, $78 \%$ ) as a colorless oil; $[\alpha]_{\mathrm{D}}{ }^{23}-19.5^{\circ}\left(c 0.43, \mathrm{CHCl}_{3}\right)$; IR (film) $3290,3028,2927$, $1754,1699,1653,1558,1497,1456,1399,1093,918 \mathrm{~cm}^{-1} ;{ }^{1} \mathrm{H}$ NMR $(500 \mathrm{MHz}$, $\left.\mathrm{CDCl}_{3}\right) \delta 7.39-7.13(\mathrm{~m}, 8 \mathrm{H}), 6.91(\mathrm{dd}, J=7.2 \mathrm{~Hz}, 2 \mathrm{H}), 5.74$ (dddd, $J=10.2,10.2$, 7.8, $6.1 \mathrm{~Hz}, 1 \mathrm{H}), 5.13(\mathrm{dd}, J=17.1,1.4 \mathrm{~Hz}, 1 \mathrm{H}), 5.05(\mathrm{dd}, J=10.3,1.0 \mathrm{~Hz}, 1 \mathrm{H}), 4.43(\mathrm{~s}, 1 \mathrm{H}), 4.23(\mathrm{ddd}$, 
$J=7.9,1.3,1.3 \mathrm{~Hz}, 1 \mathrm{H}), 3.77-3.70(\mathrm{~m}, 2 \mathrm{H}), 3.16(\mathrm{dd}, J=13.4,3.4 \mathrm{~Hz}, 1 \mathrm{H}), 3.06-3.04(\mathrm{~m}, 1 \mathrm{H}), 2.45-$ $2.40(\mathrm{~m}, 3 \mathrm{H}) ;{ }^{13} \mathrm{C} \mathrm{NMR}\left(125 \mathrm{MHz}, \mathrm{CDCl}_{3}\right) \delta 157.7,142.1,135.99,135.98,134.2,129.1,128.8,128.5$, 127.98, 127.93, 126.9, 118.4, 65.8, 62.9, 58.2, 40.3, 36.8; MS (EI) $\mathrm{m} / z$ (relative intensity) $323\left([\mathrm{M}+\mathrm{H}]^{+}\right.$, $40 \%$ ); Anal. Calcd. for $\mathrm{C}_{20} \mathrm{H}_{22} \mathrm{~N}_{2} \mathrm{O}_{2}:$ C, 74.50; H, 6.88; N, 8.69. Found: C, 74.40; H, 6.89; N, 8.59.

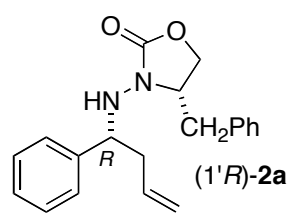

(4S,1'R)-3-(1'-Allyl-1'-phenylmethyl-amino)-4-phenylmethyl-2oxazolidinone ((1'R)-2a). From $1 \mathbf{a}(84 \mathrm{mg}, 0.30 \mathrm{mmol})$ by General Procedure B was obtained $2 \mathbf{a}(54 \mathrm{mg}, 56 \%)$ as a mixture of diastereomers $\left(\left(1^{\prime} S\right):\left(1^{\prime} R\right)=5: 1\right)$, along with unreacted 1a $(25 \mathrm{mg}, 30 \%)$. From a larger scale run (302 mg of 1a, $1.08 \mathrm{mmol})$ was obtained a mixture of (1'S)-2a and (1'R)-2a (107 mg, 33\%). Fractions enriched in the minor diastereomer were pooled and further flash chromatography afforded pure $\left(1^{\prime} R\right)-\mathbf{2 a}(10 \mathrm{mg}$, $3 \%$ ) as a colorless solid. $\mathrm{mp} 63-64{ }^{\circ} \mathrm{C}$; $[\alpha]_{\mathrm{D}}{ }^{20}+60^{\circ}\left(c 0.08, \mathrm{CHCl}_{3}\right)$; IR (film) 3277, 3030, 2916, 1759, 1699, 1653, 1559, 1507, 1457, $1091 \mathrm{~cm}^{-1} ;{ }^{1} \mathrm{H}$ NMR $\left(500 \mathrm{MHz}, \mathrm{CDCl}_{3}\right) \delta$ 7.40-7.22 (m, 8H), $7.02(\mathrm{dd}, J$ $=7.4 \mathrm{~Hz}, 2 \mathrm{H}), 5.79(\mathrm{dddd}, J=10.3,10.3,6.5,6.5 \mathrm{~Hz}, 1 \mathrm{H}), 5.14(\mathrm{dd}, J=17.0,1.4 \mathrm{~Hz}, 1 \mathrm{H}), 5.07(\mathrm{dd}, J=$ $10.2 \mathrm{~Hz}, 1 \mathrm{H}), 4.38(\mathrm{~d}, J=1.5 \mathrm{~Hz}, 1 \mathrm{H}), 4.30(\mathrm{ddd}, J=6.9,1.5,1.5 \mathrm{~Hz}, 1 \mathrm{H}), 4.02(\mathrm{dd}, J=7.8,7.6 \mathrm{~Hz}$, $1 \mathrm{H}), 3.87-3.80(\mathrm{~m}, 2 \mathrm{H}), 2.95(\mathrm{dd}, J=13.8,3.5 \mathrm{~Hz}, 1 \mathrm{H}), 2.58-2.53(\mathrm{dd}, J=7.9,7.7 \mathrm{~Hz}, 2 \mathrm{H}), 2.00(\mathrm{dd}, J=$ $10.3,9.8 \mathrm{~Hz}, 1 \mathrm{H}) ;{ }^{13} \mathrm{C}$ NMR $\left(125 \mathrm{MHz}, \mathrm{CDCl}_{3}\right) \delta 159.0,140.9,136.1,134.6,134.2,129.1,128.9,128.5$, $128.4,127.98,126.98,118.1,66.5,62.9,59.4,39.4,37.4$; MS (EI) $\mathrm{m} / z$ (relative intensity) 281 ([Mallyl] $]^{+}, 100 \%$ ); Anal. Calcd. for $\mathrm{C}_{20} \mathrm{H}_{22} \mathrm{~N}_{2} \mathrm{O}_{2}$ : C, 74.50; H, 6.88; N, 8.69. Found: C, 74.70; H, 7.08; N, 8.43 .

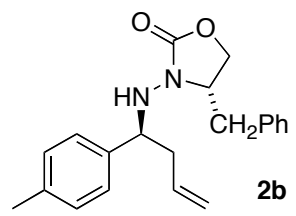

(4S,1'S)-3-(1'-Allyl-1'-(4''-methyl)-phenylmethyl-amino)-4-phenylmethyl-2oxazolidinone (2b). From $\mathbf{1 b}(59 \mathrm{mg}, 0.20 \mathrm{mmol})$ by General Procedure D was obtained $\mathbf{2 b}(63 \mathrm{mg}, 94 \%)$ as a colorless oil; $[\alpha]_{\mathrm{D}}{ }^{20}-27^{\circ}\left(c 0.1, \mathrm{CHCl}_{3}\right)$; IR (film) 3286, 3026, 2918, 1757, 1638, 1604, 1497, 1454, 1237, 1092, $918 \mathrm{~cm}^{-1} ;{ }^{1} \mathrm{H} \mathrm{NMR}$ $\left(500 \mathrm{MHz}, \mathrm{CDCl}_{3}\right) \delta$ 7.33-7.17 (m, 8H), $7.00(\mathrm{dd}, J=7.0,7.0 \mathrm{~Hz}, 2 \mathrm{H}), 5.82$ (dddd, $J$ $=17.3,10.3,7.7,6.1 \mathrm{~Hz}, 1 \mathrm{H}), 5.20(\mathrm{dd}, J=17.1,1.4 \mathrm{~Hz}, 1 \mathrm{H}), 5.12(\mathrm{dd}, J=10.2,1.8 \mathrm{~Hz}, 1 \mathrm{H}), 4.47(\mathrm{~s}$, $1 \mathrm{H}), 4.26(\mathrm{dd}, J=7.0,7.0 \mathrm{~Hz}, 1 \mathrm{H}), 3.83-3.81(\mathrm{~m}, 2 \mathrm{H}), 3.22(\mathrm{dd}, J=13.3,3.3 \mathrm{~Hz}, 1 \mathrm{H}), 3.19-3.16(\mathrm{~m}$, $1 \mathrm{H}), 2.52-2.45(\mathrm{~m}, 3 \mathrm{H}), 2.36(\mathrm{~s}, 3 \mathrm{H}) ;{ }^{13} \mathrm{C} \mathrm{NMR}\left(125 \mathrm{MHz}, \mathrm{CDCl}_{3}\right) \delta 157.8,139.0,137.6,136.2,134.4$, 129.3, 128.9, 127.9, 127.0, 118.4, 65.8, 62.6, 58.3, 40.4, 36.7, 21.3; MS (CI) $\mathrm{m} / z$ (relative intensity) 337 $\left([\mathrm{M}+\mathrm{H}]^{+}, 4 \%\right)$.

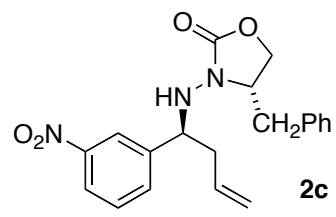

(4S,1'S)-3-(1'-Allyl-1'-(3'’-nitro)phenylmethyl-amino)-4-phenylmethyl-2oxazolidinone (2c). From 1c $(80 \mathrm{mg}, 0.25 \mathrm{mmol})$ by General Procedure D was obtained 2c (64 mg, 71\%) as a colorless solid; mp $47-48{ }^{\circ} \mathrm{C} ;[\alpha]_{\mathrm{D}}{ }^{23}-46^{\circ}(c 0.31$, $\mathrm{CHCl}_{3}$ ); IR (film) 3291, 3064, 2917, 1756, 1642, 1531, 1351, 1267, 1096, 1002 $\mathrm{cm}^{-1} ;{ }^{1} \mathrm{H} \mathrm{NMR}\left(500 \mathrm{MHz}, \mathrm{CDCl}_{3}\right) \delta 8.39(\mathrm{~s}, 1 \mathrm{H}), 8.16(\mathrm{ddd}, J=2.2,2.2,1.2 \mathrm{~Hz}$, 1H), 7.68-7.05 (m, 7H), 5.78 (dddd, $J=10.1,10.1,7.9,6.2 \mathrm{~Hz}, 1 \mathrm{H}), 5.12-5.04(\mathrm{~m}, 2 \mathrm{H}), 4.52(\mathrm{~s}, 1 \mathrm{H})$, $4.46(\mathrm{dd}, J=7.5,7.5 \mathrm{~Hz}, 1 \mathrm{H}), 3.91(\mathrm{dd}, J=9.0,4.0 \mathrm{~Hz}, 1 \mathrm{H}), 3.76-3.70(\mathrm{~m}, 1 \mathrm{H}), 3.19-3.11(\mathrm{~m}, 2 \mathrm{H}), 2.57$ $(\mathrm{dd}, J=13.0,9.6 \mathrm{~Hz}, 1 \mathrm{H}), 2.38-2.28(\mathrm{~m}, 2 \mathrm{H}) ;{ }^{13} \mathrm{C} \mathrm{NMR}\left(125 \mathrm{MHz}, \mathrm{CDCl}_{3}\right) \delta 157.9,144.6,135.6,134.3$, 133.1, 129.5, 129.2, 129.0, 127.2, 122.9, 122.4, 119.4, 65.8, 62.4, 58.9, 40.6, 36.9; MS (EI) $\mathrm{m} / z$ (relative intensity) $368\left([\mathrm{M}+\mathrm{H}]^{+}, 42 \%\right)$; Anal. Calcd. for $\mathrm{C}_{20} \mathrm{H}_{21} \mathrm{~N}_{3} \mathrm{O}_{4}: \mathrm{C}, 65.38 ; \mathrm{H}, 5.76 ; \mathrm{N}, 11.44$. Found: C, $65.31 ; \mathrm{H}, 5.79 ; \mathrm{N}, 11.33$. 


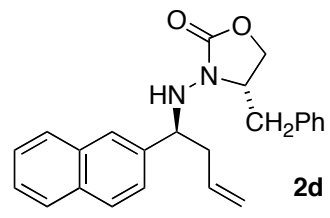
$1 \mathrm{H}), 5.21$ (dddd, $J=17.0,1.5,1.4,1.5 \mathrm{~Hz}, 1 \mathrm{H}), 5.13(\mathrm{dd}, \mathrm{J}=10.2,1.8 \mathrm{~Hz}, 1 \mathrm{H}), 4.57(\mathrm{dd}, J=1.4,1.4$ $\mathrm{Hz}, 1 \mathrm{H}), 4.47(\mathrm{ddd}, J=7.9,7.9,1.3 \mathrm{~Hz}, 1 \mathrm{H}), 3.78(\mathrm{dd}, \mathrm{J}=8.9,4.3 \mathrm{~Hz}, 1 \mathrm{H}), 3.69(\mathrm{dd}, J=8.8,7.9 \mathrm{~Hz}$, $1 \mathrm{H}), 3.25(\mathrm{dd}, J=13.4,3.3 \mathrm{~Hz}, 1 \mathrm{H}), 3.07$ (ddd, $J=13.7,7.7,3.9,3.9 \mathrm{~Hz}, 1 \mathrm{H}), 2.58-2.48(\mathrm{~m}, 3 \mathrm{H}) ;{ }^{13} \mathrm{C}$ NMR $\left(125 \mathrm{MHz}, \mathrm{CDCl}_{3}\right) \delta 157.8,139.5,135.91,135.9,134.1,133.3,133.2,129.1,128.8,128.4,127.9$, $127.8,127.4,126.9,126.4,126.1,125.3,118.6,65.7,63.0,58.3,40.4,36.8 ; \mathrm{MS}$ (EI) $\mathrm{m} / z$ (relative intensity) $372\left([\mathrm{M}+\mathrm{H}]^{+}\right.$, 4\%); Anal. Calcd. for $\mathrm{C}_{24} \mathrm{H}_{24} \mathrm{~N}_{2} \mathrm{O}_{2}$ : C, 77.39; H, 6.49; N, 7.52. Found: C, 77.07; $\mathrm{H}, 6.81 ; \mathrm{N}, 7.18$.

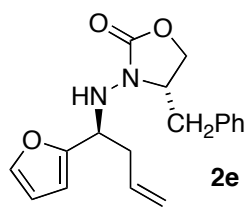

(4S,1'S)-3-(1'-Allyl-1'-furanyl-methyl-amino)-4-phenylmethyl-2-oxazolidinone (2e). From 1e (60 mg, $0.20 \mathrm{mmol})$ by General Procedure D was obtained $2 \mathrm{e}(36 \mathrm{mg}$, $58 \%$ ) as a colorless oil; $[\alpha]_{\mathrm{D}}^{20}-13^{\circ}\left(c 0.1, \mathrm{CHCl}_{3}\right)$; IR (film) 3286, 3064, 3028, 2917, 1756, 1643, 1558, 1497, 1406, 1239, $1094 \mathrm{~cm}^{-1} ;{ }^{1} \mathrm{H}$ NMR $\left(500 \mathrm{MHz}, \mathrm{CDCl}_{3}\right) \delta 7.44-$ $7.04(\mathrm{~m}, 6 \mathrm{H}), 6.35(\mathrm{dd}, J=3.2,2.2 \mathrm{~Hz}, 1 \mathrm{H}), 6.27(\mathrm{dd}, J=3.1,1.5 \mathrm{~Hz}, 1 \mathrm{H}), 5.77$ (dddd, $J=10.2,10.2,7.8,6.1 \mathrm{~Hz}, 1 \mathrm{H}), 5.20(\mathrm{dddd}, J=17.1,1.4,1.4,1.4 \mathrm{~Hz}, 1 \mathrm{H}), 5.13(\mathrm{dd}, J=9.8,0.6 \mathrm{~Hz}$, $1 \mathrm{H}), 4.41-4.38(\mathrm{~m}, 2 \mathrm{H}), 3.97-3.89(\mathrm{~m}, 2 \mathrm{H}), 3.22$ (dddd, $J=15.7,3.8,3.8,3.8 \mathrm{~Hz}, 1 \mathrm{H}), 2.62-2.55(\mathrm{~m}$, $3 \mathrm{H}) ;{ }^{13} \mathrm{C} \mathrm{NMR}\left(125 \mathrm{MHz}, \mathrm{CDCl}_{3}\right) \delta 157.7,154.1,142.3,135.9,133.7,129.3,128.9,126.9,118.5,110.6$, 108.5, 66.1, 58.3, 56.0, 36.9; MS (CI) $\mathrm{m} / z$ (relative intensity) 313 ([M+H] $]^{+}, 0.8 \%$ ); Anal. Calcd. for $\mathrm{C}_{18} \mathrm{H}_{20} \mathrm{~N}_{2} \mathrm{O}_{3}: \mathrm{C}, 69.21 ; \mathrm{H}, 6.45 ; \mathrm{N}, 8.97$. Found: $\mathrm{C}, 69.39 ; \mathrm{H}, 6.54 ; \mathrm{N}, 8.75$.

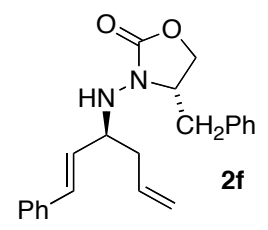

(4S,1'S)-3-(1'-Allyl-1'-trans-cinnamyl-methyl-amino)-4-phenylmethyl-2-

oxazolidinone (2f). From 1 f $(66 \mathrm{mg}, 0.22 \mathrm{mmol})$ by General Procedure D was obtained 2f $(45 \mathrm{mg}, 60 \%)$ as a colorless oil; $[\alpha]_{\mathrm{D}}^{23}-48.7^{\circ}\left(c 0.11, \mathrm{CHCl}_{3}\right)$; IR (film) 3288, 3060, 3029, 2917, 1755, 1639, 1495, 1403, 1266, 1093, $970 \mathrm{~cm}^{-1} ;{ }^{1} \mathrm{H}$ NMR $(500$ $\left.\mathrm{MHz} \mathrm{CDCl}_{3}\right) \delta 7.42-7.25(\mathrm{~m}, 8 \mathrm{H}), 7.12(\mathrm{dd}, J=7.9 \mathrm{~Hz}, 2 \mathrm{H}), 6.56(\mathrm{dd}, J=15.8 \mathrm{~Hz}$, $1 \mathrm{H}), 6.09$ (ddd, $J=15.9,8.7,1.2 \mathrm{~Hz}, 1 \mathrm{H}), 5.86(\mathrm{dddd}, J=17.3,10.4,7.0,7.0 \mathrm{~Hz}, 1 \mathrm{H}), 5.21$ (ddd, $J=$ 2.9, 2.9, $1.4 \mathrm{~Hz}, 1 \mathrm{H}), 5.15(\mathrm{dd}, J=10.3 \mathrm{~Hz}, 1 \mathrm{H}), 4.28(\mathrm{~s}, 1 \mathrm{H}), 4.05(\mathrm{dd}, J=7.7,7.7 \mathrm{~Hz}, 1 \mathrm{H}), 3.96-3.89$ (m, 3H), $3.29(\mathrm{dd}, J=13.5,3.6 \mathrm{~Hz}, 1 \mathrm{H}), 2.65(\mathrm{dd}, \mathrm{J}=13.8,9.5 \mathrm{~Hz}, 1 \mathrm{H}), 2.36(\mathrm{dd}, J=6.8,5.8 \mathrm{~Hz}, 2 \mathrm{H})$; ${ }^{13} \mathrm{C}$ NMR $\left(125 \mathrm{MHz}, \mathrm{CDCl}_{3}\right) \delta 157.9,136.8,135.9,133.9,133.3,130.3,129.3,128.9,128.8,127.8$, 127.1, 126.6, 118.3, 66.0, 61.7, 59.5, 38.5, 37.3; MS (CI) $\mathrm{m} / z$ (relative intensity) $349\left([\mathrm{M}+\mathrm{H}]^{+}, 3 \%\right)$; Anal. Calcd. for $\mathrm{C}_{22} \mathrm{H}_{24} \mathrm{~N}_{2} \mathrm{O}_{2}:$ C, 75.83; H, 6.94; N, 8.04. Found: C, 75.61; H, 6.95; N, 7.97.

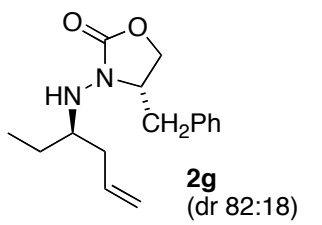

(4S, 3R)-3-(5'-Hexen-3'-yl)amino)-4-phenylmethyl-2-oxazolidinone (2g). From $1 \mathrm{~g}(50 \mathrm{mg}, 0.20 \mathrm{mmol})$ by General Procedure D was obtained $2 \mathrm{~g}(28 \mathrm{mg}, 51 \%$, dr 82:18) as a colorless oil; IR (film) 3290, 3028, 2965, 2918, 1759, 1637, 1497, 1455, 1399, 1217, $1090 \mathrm{~cm}^{-1}$; Major diastereomer: ${ }^{1} \mathrm{H} \mathrm{NMR}\left(500 \mathrm{MHz}, \mathrm{CDCl}_{3}\right) \delta$ $7.32-7.13(\mathrm{~m}, 5 \mathrm{H}), 5.88$ (dddd, $J=10.2,10.2,7.8,6.2 \mathrm{~Hz}, 1 \mathrm{H}), 5.18$ (ddd, $J=17.1$, $3.4,1.6 \mathrm{~Hz}), 5.13(\mathrm{dd}, J=6.5,1.2 \mathrm{~Hz}, 1 \mathrm{H}), 4.13(\mathrm{dd}, J=8.9,4.5 \mathrm{~Hz}, 1 \mathrm{H}), 4.00-3.98(\mathrm{~m}, 1 \mathrm{H}), 3.95-3.86$ (m, 2H), 3.30 (ddd, $J=13.1,3.4,3.4 \mathrm{~Hz}, 1 \mathrm{H}), 2.30-2.21(\mathrm{~m}, 3 \mathrm{H}), 1.51-1.48(\mathrm{~m}, 2 \mathrm{H}), 0.98-0.90(\mathrm{~m}, 3 \mathrm{H})$; ${ }^{13} \mathrm{C} \mathrm{NMR}\left(125 \mathrm{MHz}, \mathrm{CDCl}_{3}\right) \delta$ 158.4, 136.1, 135.0, 129.1, 128.9, 127.1, 117.6, 65.9, 65.7, 59.7, 59.3, 37.2, 36.3, 24.9, 9.3; MS (EI) $\mathrm{m} / \mathrm{z}$ (relative intensity) $274\left(\mathrm{M}^{+}, 0.9 \%\right.$ ); Anal. Calcd. for $\mathrm{C}_{16} \mathrm{H}_{22} \mathrm{~N}_{2} \mathrm{O}_{2}: \mathrm{C}_{\text {, }}$ 70.04; H, 8.08; N, 10.21. Found: C, 69.66; H, 8.11; N, 10.16. 


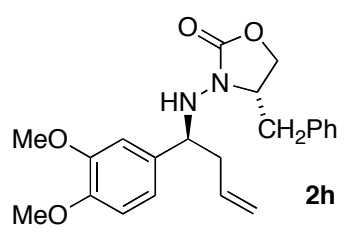

(4S,1'S)-3-(1'-(3,4-Dimethoxyphenyl)but-3'-enylamino)-4-phenylmethyl2-oxazolidinone (2h). From $\mathbf{1 h}(1.53 \mathrm{~g}, 4.5 \mathrm{mmol})$ by General Procedure D, using $\operatorname{In}(\mathrm{OTf})_{3}$ from Alfa Aesar, was obtained $\mathbf{2 h}(920 \mathrm{mg}$, dr $96: 4,54 \%$ yield $)$ as a colorless oil. Major diastereomer (1'S)-2h: $[\alpha]_{\mathrm{D}}^{23}-75^{\circ}\left(c 0.08, \mathrm{CHCl}_{3}\right)$; IR (film) 3286, 2934, 2835, 1753, 1593, 1516, 1455, 1262, 1237, 1141, $1092 \mathrm{~cm}^{-1}$; ${ }^{1} \mathrm{H}$ NMR $\left(500 \mathrm{MHz}, \mathrm{CDCl}_{3}\right) \delta 7.25-7.18(\mathrm{~m}, 7 \mathrm{H}), 6.83(\mathrm{~d}, J=8.1 \mathrm{~Hz}, 1 \mathrm{H}), 5.81-5.75(\mathrm{~m}, 1 \mathrm{H}), 5.17(\mathrm{~d}, J$ $=17.0 \mathrm{~Hz}, 1 \mathrm{H}), 5.10(\mathrm{~d}, J=10.2 \mathrm{~Hz}, 1 \mathrm{H}), 4.45(\mathrm{br} \mathrm{s}, 1 \mathrm{H}), 4.24(\mathrm{dd}, J=7.1,7.1 \mathrm{~Hz}, 1 \mathrm{H}), 3.88(\mathrm{~s}, 3 \mathrm{H})$, $3.86(\mathrm{~s}, 3 \mathrm{H}), 3.80-3.79(\mathrm{~m}, 2 \mathrm{H}), 3.21-3.15(\mathrm{~m}, 2 \mathrm{H}), 2.51(\mathrm{dd}, J=13.1,9.5 \mathrm{~Hz}, 1 \mathrm{H}), 2.46-2.43(\mathrm{~m}, 2 \mathrm{H})$; ${ }^{13} \mathrm{C}$ NMR $\left(125 \mathrm{MHz}, \mathrm{CDCl}_{3}\right) \delta 157.6,149.1,148.7,135.9,134.5,134.3,129.0,128.8,126.9,120.3$, $118.3,111.1,110.8,65.8,62.4,58.1,56.0,55.9,40.3,36.8 ; \mathrm{MS}$ (APCI) $\mathrm{m} / z$ (relative intensity) 383 $\left([\mathrm{M}+\mathrm{H}]^{+}, 7 \%\right)$; Anal. Calcd. for $\mathrm{C}_{22} \mathrm{H}_{26} \mathrm{~N}_{2} \mathrm{O}_{4}: \mathrm{C}, 69.10 ; \mathrm{H}, 6.85 ; \mathrm{N}, 7.32$. Found: C, 68.91; H, 6.75; N, 7.30. Minor diastereomer (1'R)-2h: Colorless oil; $[\alpha]_{\mathrm{D}}^{23}+28^{\circ}\left(c 0.1, \mathrm{CHCl}_{3}\right)$; IR (film) 3281, 2934, 2836, 1754, 1593, 1516, 1455, 1419, 1263, 1141, 1092, $1027 \mathrm{~cm}^{-1} ;{ }^{1} \mathrm{H} \mathrm{NMR}\left(500 \mathrm{MHz}, \mathrm{CDCl}_{3}\right) \delta 7.27-$ $7.03(\mathrm{~m}, 5 \mathrm{H}), 6.95(\mathrm{~d}, J=1.9 \mathrm{~Hz}, 1 \mathrm{H}), 6.89(\mathrm{~d}, J=8.2 \mathrm{~Hz}, 1 \mathrm{H}), 6.80(\mathrm{~d}, J=8.2 \mathrm{~Hz}, 1 \mathrm{H}), 5.71(\mathrm{dddd}, J=$ $16.3,13.8,7.3,6.1 \mathrm{~Hz}, 1 \mathrm{H}), 5.14-5.12(\mathrm{~m}, 1 \mathrm{H}), 5.07-5.02(\mathrm{~m}, 1 \mathrm{H}), 4.40-4.25(\mathrm{br}, 1 \mathrm{H}), 4.20(\mathrm{dd}, J=7.0$, $7.0 \mathrm{~Hz}, 1 \mathrm{H}), 4.01(\mathrm{dd}, J=7.3,7.3 \mathrm{~Hz}, 1 \mathrm{H}), 3.88-3.82(\mathrm{~m}, 2 \mathrm{H}), 3.87(\mathrm{~s}, 3 \mathrm{H}), 3.84(\mathrm{~s}, 3 \mathrm{H}), 3.00(\mathrm{dd}, J=$ $13.9,3.8 \mathrm{~Hz}, 1 \mathrm{H}), 2.52-2.43(\mathrm{~m}, 2 \mathrm{H}), 2.14(\mathrm{dd}, J=13.0,10.0 \mathrm{~Hz}, 1 \mathrm{H}) ;{ }^{13} \mathrm{C} \mathrm{NMR}\left(125 \mathrm{MHz}, \mathrm{CDCl}_{3}\right) \delta$ $158.7,149.0,148.8,136.0,134.6,133.4,128.9,128.8,127.0,120.5,117.9,111.3,111.0,66.4,62.5$, 58.9, 56.0, 55.9, 39.7, 37.6; MS (APCI) $\mathrm{m} / \mathrm{z}$ (relative intensity) 341 ([M-allyl $]^{+}, 100 \%$ ); Anal. Calcd. for $\mathrm{C}_{22} \mathrm{H}_{26} \mathrm{~N}_{2} \mathrm{O}_{4}$ : C, 69.10; H, 6.85; N, 7.32. Found: C, 68.83; H, 6.84; N, 7.08.

Trifluoroacetyl-mediated N-N bond cleavage with $\mathrm{SmI}_{2}$ (General Procedure E): To a solution of the hydrazone in THF $(0.1 \mathrm{M})$ at $-78^{\circ} \mathrm{C}$ was added $n$-BuLi $(1.6 \mathrm{M}$ in hexane, 1.1 equiv). After $40 \mathrm{~min}$, freshly distilled trifluoroacetic anhydride (1.8 equiv) was added and the mixture was allowed to warm to ambient temperature over $16 \mathrm{~h}$. Concentration and flash chromatography gave the trifluoroacetamide. To a solution of the trifluoroacetamide in $\mathrm{MeOH}(0.5 \mathrm{M})$, was added $\mathrm{SmI}_{2}(0.3 \mathrm{M}$ in THF, 8 equiv) dropwise under $\mathrm{N}_{2}$ via a syringe. After $30 \mathrm{~min}$, the dark blue solution was opened to air, and the color changed to yellow. Concentration and flash chromatography gave the amide products and recovered auxiliary where applicable. Note the concentration of $\mathrm{SmI}_{2}$ was assumed on the basis of preparation; the actual concentration is likely lower than $0.3 \mathrm{M}$. Use of a freshly titrated $\mathrm{SmI}_{2}$ solution $(0.1 \mathrm{M})$ showed a 3:1 molar ratio of $\mathrm{SmI}_{2}$ : substrate was sufficient to achieve complete conversion in $5 \mathrm{~min}$.

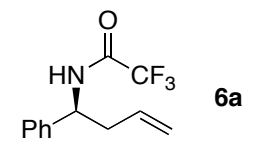

(S)-2,2,2-Trifluoro- $N$-(1-phenyl-but-3-enyl)-acetamide (6a). From 2a (10 mg, $0.03 \mathrm{mmol}$ ) by General Procedure E was obtained the $N$-TFA derivative (11 $\mathrm{mg}, 85 \%)$ as colorless oil; IR (film) 1795, 1713, 1702, $1650 \mathrm{~cm}^{-1}$; MS (APCI) $\mathrm{m} / z$ (relative intensity) $419\left([\mathrm{M}+1]^{+}, 57 \%\right)$. Treatment of this trifluoroacetamide (17 $\mathrm{mg}, 0.041$ mmol) with $\mathrm{SmI}_{2}$ by General Procedure E was obtained 6a $(9.4 \mathrm{mg}, 95 \%)$ as colorless solid: $\mathrm{mp} 70-72$ ${ }^{\circ} \mathrm{C} ;[\alpha]_{\mathrm{D}}{ }^{25}-102^{\circ}\left(c 0.9, \mathrm{CHCl}_{3}\right.$ ); IR (film) 3343, 3080, 2950, 2924, 2857, 1696, 1543, 1176, $926 \mathrm{~cm}^{-1} ;{ }^{1} \mathrm{H}$ NMR (500 MHz, $\left.\mathrm{CDCl}_{3}\right) \delta$ 7.37-7.24 (m, 5H), 6.57 (br, 1H), 5.67 (dddd, $J=17.5,10.5,7.5,7.5 \mathrm{~Hz}$, $1 \mathrm{H}), 5.17-5.12(\mathrm{~m}, 2 \mathrm{H}), 5.07$ (dddd, apparent quintet, $J=7.0 \mathrm{~Hz}, 1 \mathrm{H}), 2.64(\mathrm{dd}, J=6.9,6.9 \mathrm{~Hz}, 2 \mathrm{H}) ;{ }^{13} \mathrm{C}$ $\operatorname{NMR}\left(125 \mathrm{MHz}, \mathrm{CDCl}_{3}\right) \delta 156.5\left(\mathrm{~d},{ }^{2} J_{\mathrm{CF}}=36.9 \mathrm{~Hz}\right), 139.5,132.8,128.9,128.1,126.4,119.3,115.9(\mathrm{~d}$, $\left.{ }^{1} J_{\mathrm{CF}}=286.4 \mathrm{HZ}\right), 53.2,39.9$; MS (CI) $\mathrm{m} / \mathrm{z}$ (relative intensity) $244\left([\mathrm{M}+\mathrm{H}]^{+}, 3 \%\right)$; Anal. Calcd. for $\mathrm{C}_{12} \mathrm{H}_{12} \mathrm{~F}_{3} \mathrm{NO}$ : C, 59.26; H, 4.97; N, 5.76. Found: C, 59.24; H, 4.94; N, 5.61.

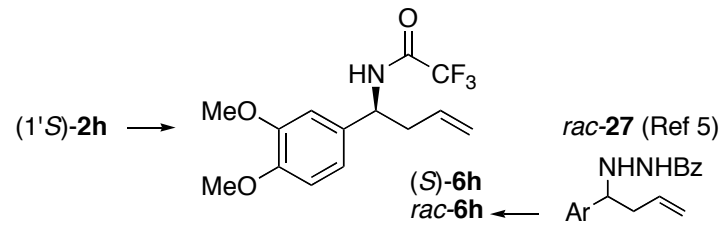

N-[1-(3,4-Dimethoxy-phenyl)-but-3-enyl]-2,2,2trifluoroacetamide (6h). From $2 \mathbf{h}(0.907 \mathrm{~g}, 2.37 \mathrm{mmol})$ by General Procedure E was obtained the $N$-TFA derivative $(1.01 \mathrm{~g}, 89 \%)$ as colorless oil; IR (film) 1795 , 
1714, 1700, $1649 \mathrm{~cm}^{-1}$; MS (CI) m/z (relative intensity) $479\left([\mathrm{M}+1]^{+}, 18 \%\right)$. Treatment of this trifluoroacetamide (964 mg, $2.0 \mathrm{mmol})$ with $\mathrm{SmI}_{2}$ by General Procedure E was obtained $(S)-6 \mathbf{h}(575 \mathrm{mg}$, 94\%) as colorless solid; $[\alpha]_{\mathrm{D}}{ }^{25}-64^{\circ}\left(c 0.17, \mathrm{CHCl}_{3}\right)$. This compound was spectroscopically $\left({ }^{1} \mathrm{H}\right.$ and ${ }^{13} \mathrm{C}$ NMR) identical to a racemic sample prepared from $27^{5}(159 \mathrm{mg}, 0.489 \mathrm{mmol})$ by treatment with $\mathrm{SmI}_{2}$ as described within General Procedure E, giving amine rac-7 (96 mg, 97\%) as a colorless solid. To a solution of rac-7 $(0.20 \mathrm{~g}, 0.966 \mathrm{mmol})$ in anhydrous $\mathrm{CH}_{2} \mathrm{Cl}_{2}(3 \mathrm{~mL})$ was added $\left(\mathrm{CF}_{3} \mathrm{CO}\right)_{2} \mathrm{O}$ (redistilled, $0.28 \mathrm{~mL}, 1.96 \mathrm{mmol}$ ) dropwise via syringe. After ca. $16 \mathrm{~h}$, the resulting yellow mixture was directly submitted to flash chromatography (2:1 hexane/EtOAc) to give rac-6h $(276 \mathrm{mg}, 95 \%)$ as a colorless solid; mp 54-56 ${ }^{\circ} \mathrm{C}$; IR (film) 3305, 3082, 2938, 2839, 1700, 1518, 1266, 1183, 1161, $1027 \mathrm{~cm}^{-1} ;{ }^{1} \mathrm{H}$ $\operatorname{NMR}\left(500 \mathrm{MHz}, \mathrm{CDCl}_{3}\right) \delta$ 6.84-6.80 (m, 2H), $6.76(\mathrm{~s}, 1 \mathrm{H}), 6.45(\mathrm{br} \mathrm{d}, J=5.9 \mathrm{~Hz}, 1 \mathrm{H}), 5.67$ (dddd, $J=$ $17.1,10.2,7.0,7.0 \mathrm{~Hz}, 1 \mathrm{H}), 5.17-5.11(\mathrm{~m}, 2 \mathrm{H}), 5.00(\mathrm{ddd}, J=7.1,7.1,7.1 \mathrm{~Hz}, 1 \mathrm{H}), 3.86(\mathrm{~s}, 3 \mathrm{H}), 3.85$ $(\mathrm{s}, 3 \mathrm{H}), 2.62(\mathrm{dd}, J=7.0,7.0 \mathrm{~Hz}, 2 \mathrm{H}) ;{ }^{13} \mathrm{C} \mathrm{NMR}\left(125 \mathrm{MHz}, \mathrm{CHCl}_{3}\right) \delta 156.4\left({ }^{2} J_{\mathrm{CF}}=36.9 \mathrm{~Hz}\right), 149.4$, 149.0, 133.0, 132.0, 119.0, 118.5, $115.9\left(\mathrm{~d},{ }^{1} J_{\mathrm{CF}}=286.5 \mathrm{~Hz}\right), 111.5,110.2,56.0(2 \mathrm{C}), 53.1,39.7 ;{ }^{13} \mathrm{C}$ NMR $\left(125 \mathrm{MHz}\right.$, toluene- $\left.d_{8}\right) \delta 156.0\left(\mathrm{~d},{ }^{2} J_{\mathrm{CF}}=46.4 \mathrm{~Hz}\right), 150.1,149.7,133.5,132.3,118.5,118.1,116.4$ $\left({ }^{1} J_{\mathrm{CF}}=285.3 \mathrm{~Hz}\right), 112.2,111.1,55.4,55.3,53.0,39.3$; MS (APCI) $\mathrm{m} / z$ (relative intensity) $304\left([\mathrm{M}+\mathrm{H}]^{+}\right.$, $6 \%$ ); Anal. Calcd. for $\mathrm{C}_{12} \mathrm{H}_{12} \mathrm{~F}_{3} \mathrm{NO}$ : C, 55.44; H, 5.32; N, 4.62. Found: C, 55.55; H, 5.36; N, 4.70.

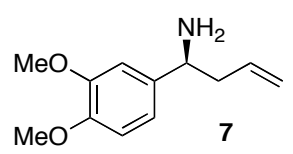

(S)-1-(3,4-Dimethoxyphenyl)-but-3-enylamine (7). To a solution of $6 \mathbf{h}(0.18 \mathrm{~g}$, $0.594 \mathrm{mmol})$ in $\mathrm{MeOH} / \mathrm{H}_{2} \mathrm{O}(6 \mathrm{~mL}, 5: 1)$, was added $\mathrm{K}_{2} \mathrm{CO}_{3}(0.82 \mathrm{~g}, 5.88 \mathrm{mmol})$. After heating at reflux for $3 \mathrm{~h}$, the mixture was concentrated and the residue was partitioned between EtOAc and water. The organic phase was washed with brine, dried over $\mathrm{MgSO}_{4}$ and concentrated to give $7(112 \mathrm{mg}, 91 \%)$ as pale yellow needles; mp $55-56{ }^{\circ} \mathrm{C} ;[\alpha]_{\mathrm{D}}{ }^{25}-13.6^{\circ}(c 0.5$, $\mathrm{CHCl}_{3}$ ); IR (film) 3374, 3069, 2933, 2834, 1677, 1592, 1514, 1462, 1417, 1262, 1232, 1139, $1028 \mathrm{~cm}^{-1}$; ${ }^{1} \mathrm{H}$ NMR $\left(500 \mathrm{MHz}, \mathrm{CDCl}_{3}\right) \delta$ 6.90-6.80 (m, 3H), 5.74 (dddd, $\left.J=18.1,10.2,8.0,6.2 \mathrm{~Hz}, 1 \mathrm{H}\right), 5.08(\mathrm{dd}$, $J=17.0,1.3 \mathrm{~Hz}, 1 \mathrm{H}), 5.04(\mathrm{ddd}, J=11.1,1.5,1.5 \mathrm{~Hz}, 1 \mathrm{H}), 3.95-3.88(\mathrm{~m}, 1 \mathrm{H}), 3.88(\mathrm{~s}, 3 \mathrm{H}), 3.85(\mathrm{~s}$, $3 \mathrm{H}), 2.44-2.41(\mathrm{~m}, 1 \mathrm{H}), 2.34-2.30(\mathrm{~m}, 1 \mathrm{H}), 1.65$ (br s, $2 \mathrm{H}) ;{ }^{13} \mathrm{C} \mathrm{NMR}\left(125 \mathrm{MHz}, \mathrm{CDCl}_{3}\right) \delta$ 149.1, 148.1, 138.6, 135.6, 118.4, 117.5, 111.1, 109.6, 55.9, 55.1, 44.4, 38.6; MS (CI) $\mathrm{m} / \mathrm{z}$ (relative intensity) 208 $(\mathrm{M}+1,6 \%)$.

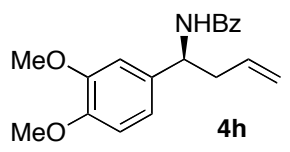

(S)- $N$-(1-(3,4-Dimethoxyphenyl)-but-3-enyl)benzamide (4h). To a solution of 2h $(1.92 \mathrm{~g}, 5.0 \mathrm{mmol})$ in THF $(90 \mathrm{~mL})$ at $-78^{\circ} \mathrm{C}$ was added $n$-BuLi $(3.2 \mathrm{~mL}, 1.6 \mathrm{M}$ in THF, $5.1 \mathrm{mmol})$. After $1 \mathrm{~h}$, benzoic anhydride $(1.4 \mathrm{~g}, 6.0 \mathrm{mmol})$ was added in one portion. The reaction mixture was allowed to warm to ambient temperature over ca. $16 \mathrm{~h}$, then partitioned between water and $\mathrm{CH}_{2} \mathrm{Cl}_{2}$. The organic phase was washed with brine and dried over $\mathrm{MgSO}_{4}$. Flash chromatography $(2: 1 \rightarrow 1: 1$ hexane/ethyl acetate) gave the benzamide as a colorless oil $(2.33 \mathrm{~g}, 96 \%)$. To a solution of this oil $(1.5 \mathrm{~g}, 3.09 \mathrm{mmol})$ in THF/HMPA $(20: 1,42 \mathrm{~mL})$ at $-78{ }^{\circ} \mathrm{C}$ was added $\mathrm{SmI}_{2}(90 \mathrm{~mL}, 0.1 \mathrm{M}$ in $\mathrm{THF}, 9.0 \mathrm{mmol})$. After $10 \mathrm{~min}$, the reaction mixture was partitioned between water and $\mathrm{CH}_{2} \mathrm{Cl}_{2}$. The organic phase was washed with brine and dried over $\mathrm{MgSO}_{4}$. Flash chromatography gave oxazolidinone $3(0.42 \mathrm{~g}, 77 \%)$ and benzamide $\mathbf{4 h}(0.78 \mathrm{~g}, 81 \%)$ as a colorless solid; mp 135-136 ${ }^{\circ} \mathrm{C}$; $[\alpha]_{\mathrm{D}}{ }^{25}-27^{\circ}\left(c 0.13, \mathrm{CHCl}_{3}\right)$; IR (film) 3323, 3069, 3002, 2940, 2909, 2832, 1633, 1520, 1462, 1264, 1232, 1142, 1025, $902 \mathrm{~cm}^{-1} ;{ }^{1} \mathrm{H}$ NMR (500 MHz, $\left.\mathrm{CDCl}_{3}\right) \delta$ 7.80-7.70 (m, 2H), 7.50$7.23(\mathrm{~m}, 3 \mathrm{H}), 6.90-6.78(\mathrm{~m}, 3 \mathrm{H}), 6.44(\mathrm{br} \mathrm{d}, J=7.5 \mathrm{~Hz}, 1 \mathrm{H}), 5.75$ (dddd, $J=17.0,10.0,7.0,7.0 \mathrm{~Hz}$, $1 \mathrm{H}), 5.20$ (ddd, $J=7.0,7.0,7.0 \mathrm{~Hz}, 1 \mathrm{H}), 5.15$ (br d, $J=17.0 \mathrm{~Hz}, 1 \mathrm{H}), 5.09$ (br d, $J=10.0 \mathrm{~Hz}, 1 \mathrm{H}), 3.83$ $(\mathrm{s}, 3 \mathrm{H}), 3.82(\mathrm{~s}, 3 \mathrm{H}), 2.70-2.62(\mathrm{~m}, 2 \mathrm{H}) ;{ }^{13} \mathrm{C} \mathrm{NMR}\left(125 \mathrm{MHz}, \mathrm{CDCl}_{3}\right) \delta 166.7,149.2,148.4,134.7$, $134.4,134.3,131.5,128.6,126.9,118.4,118.3,111.4,110.5,56.0,55.9,52.7,40.5 ; \mathrm{MS}$ (APCI) $\mathrm{m} / z$ (relative intensity) $312\left([\mathrm{M}+1]^{+}, 63 \%\right)$; Anal. Calcd. for $\mathrm{C}_{19} \mathrm{H}_{21} \mathrm{NO}_{3}: \mathrm{C}, 73.29 ; \mathrm{H}, 6.80 ; \mathrm{N}, 4.50$. Found: C, 73.36; H, 6.81; N, 4.51. 
(S)- $N$-[1-(3,4-Dimethoxyphenyl)-3-oxo-butyl]-benzamide (8). A mixture of benzamide 4 h (622 MeO $\mathrm{mg}, 2.0 \mathrm{mmol}), \mathrm{PdCl}_{2}(72 \mathrm{mg}, 0.40 \mathrm{mmol})$ and $\mathrm{Cu}(\mathrm{OAc})_{2}(148 \mathrm{mg}, 0.80 \mathrm{mmol})$ in $\mathrm{H}_{2} \mathrm{O} / \mathrm{DMF}(1: 7,16 \mathrm{~mL})$ was stirred under oxygen $(1 \mathrm{~atm})$ for $2 \mathrm{~d}$. To the brownish $\mathrm{CH}_{2} \mathrm{Cl}_{2}$. The organic phase was washed with saturated $\mathrm{NaHCO}_{3}$ solution and brine. It was dried over $\mathrm{MgSO}_{4}$, concentrated and purified by flash chromatography (1:1 hexane-EtOAc). Methyl ketone 8 was obtained as a colorless solid $(576 \mathrm{mg}, 88 \%)$. mp $139-140{ }^{\circ} \mathrm{C} ;[\alpha]_{\mathrm{D}}{ }^{21}-9.5^{\circ}\left(c 0.39, \mathrm{CHCl}_{3}\right)$; IR (film) 3329, 3064, 2997, 2940, 2837, 1715, 1636, 1516, 1462, 1418, 1359, 1261, 1142, $1026 \mathrm{~cm}^{-1}$; ${ }^{1} \mathrm{H} \mathrm{NMR}$ $\left(500 \mathrm{MHz}, \mathrm{CDCl}_{3}\right) \delta 7.78(\mathrm{~d}, J=7.0 \mathrm{~Hz}, 2 \mathrm{H}), 7.50-7.40(\mathrm{~m}, 3 \mathrm{H}), 7.29(\mathrm{~d}, J=7.8 \mathrm{~Hz}, 1 \mathrm{H}), 6.88-6.80$ $(\mathrm{m}, 3 \mathrm{H}), 5.55-5.49(\mathrm{~m}, 1 \mathrm{H}), 3.85(\mathrm{~s}, 3 \mathrm{H}), 3.83(\mathrm{~s}, 3 \mathrm{H}), 3.20(\mathrm{~d}, J=16.5 \mathrm{~Hz}, 1 \mathrm{H}), 3.00(\mathrm{~d}, J=16.5 \mathrm{~Hz}$, 1H), $2.13(\mathrm{~s}, 3 \mathrm{H}) ;{ }^{13} \mathrm{C}$ NMR $\left(125 \mathrm{MHz}, \mathrm{CDCl}_{3}\right) \delta 208.1,166.6,149.3,148.1,134.3,133.6,131.6,128.6$, 127.0, 118.4, 111.4, 110.4, 56.0, 55.9, 50.0, 48.1, 31.0; MS (APCI) $\mathrm{m} / \mathrm{z}$ (relative intensity) $327\left([\mathrm{M}+1]^{+}\right.$, 46\%); Anal. Calcd. for $\mathrm{C}_{19} \mathrm{H}_{21} \mathrm{NO}_{4}$ : C, 69.71; H, 6.47; N, 4.28. Found: C, 69.51; H, 6.36; N, 4.17.

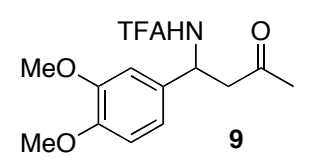

(S)-N-[1-(3,4-Dimethoxyphenyl)-3-oxo-butyl]-2,2,2-trifluoroacetamide (9). Using the procedure for preparation of $\mathbf{8}$, from trifluoroacetamide rac-6h $(45 \mathrm{mg}$, $0.14 \mathrm{mmol}), \mathrm{PdCl}_{2}(6 \mathrm{mg}, 0.03 \mathrm{mmol})$ and $\mathrm{Cu}(\mathrm{OAc})_{2}(19 \mathrm{mg}, 0.09 \mathrm{mmol})$ was obtained methyl ketone 9 (33 $\mathrm{mg}, 71 \%)$ as a colorless solid; mp 94.0-94.5 ${ }^{\circ} \mathrm{C}$; IR (KBr) 3086, 2943, 2352, 2330, 1721, 1700, 1595, 1565, 1526, 1260, 1191, 1160, $1026 \mathrm{~cm}^{-1}$; ${ }^{1} \mathrm{H}$ NMR $\left(300 \mathrm{MHz} \mathrm{CDCl}_{3}\right) \delta 7.64(\mathrm{br} \mathrm{d}, J=7.9 \mathrm{~Hz}, 1 \mathrm{H}), 6.84-6.78(\mathrm{~m}, 3 \mathrm{H}), 5.34-5.27(\mathrm{~m}, 1 \mathrm{H}), 3.86(\mathrm{~s}, 3 \mathrm{H})$, $3.84(\mathrm{~s}, 3 \mathrm{H}), 3.18(\mathrm{dd}, J=17.2,5.3 \mathrm{~Hz}, 1 \mathrm{H}), 2.97(\mathrm{dd}, J=17.2,6.1 \mathrm{~Hz}, 1 \mathrm{H}), 2.12(\mathrm{~s}, 3 \mathrm{H}) ;{ }^{13} \mathrm{C}$ NMR $(75$ $\left.\mathrm{MHz}, \mathrm{CDCl}_{3}\right) \delta 207.1,156.5\left({ }^{2} J_{\mathrm{CF}}=36.9 \mathrm{~Hz}\right), 149.2,148.9,131.6,118.4,115.7\left({ }^{1} J_{\mathrm{CF}}=286 \mathrm{~Hz}\right), 111.4$, 110.0, 55.9 (2C), 50.1, 47.0, 30.8; MS (EI) $\mathrm{m} / \mathrm{z}$ (relative intensity) $319.16\left([\mathrm{M}]^{+}, 26 \%\right.$ ); Anal. Calcd. for $\mathrm{C}_{14} \mathrm{H}_{16} \mathrm{~F}_{3} \mathrm{NO}_{4}$ : C, 52.67; H, 5.05; N, 4.39. Found: C, 52.57; H, 4.91; N, 4.33.

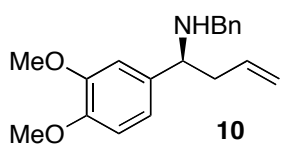

(S)-Benzyl-[1-(3,4-dimethoxy-phenyl)-but-3-enyl]amine (10). To a solution of benzamide $4 \mathbf{h}(227 \mathrm{mg}, 0.73 \mathrm{mmol})$ in THF $(7.0 \mathrm{~mL})$ was added $\mathrm{LiAlH}_{4}(1.0 \mathrm{M}$ in $\left.\mathrm{Et}_{2} \mathrm{O}, 3.0 \mathrm{~mL}, 3.0 \mathrm{mmol}\right)$. The mixture was heated at reflux for $20 \mathrm{~h}$, after which the mixture was cloudy white, then was quenched by saturated $\mathrm{NH}_{4} \mathrm{Cl}$ solution. The resulting slurry was diluted with EtOAc and filtered with the aid of additional EtOAc. The filtrate was dried over $\mathrm{MgSO}_{4}$, concentrated and purified by flash chromatography (2:1 Hexane- EtOAc) to give the benzylamine 10 $(152 \mathrm{mg}, 70 \%)$ as a colorless oil; $[\alpha]_{\mathrm{D}}{ }^{25}-55^{\circ}\left(c 1.0, \mathrm{CHCl}_{3}\right)$; IR (film) 3323, 3067, 2999, 2933, 2833, $1638,1592,1514,1463,1418,1359,1263,1234,1157,1140,1029,916 \mathrm{~cm}^{-1} ;{ }^{1} \mathrm{H}$ NMR $(500 \mathrm{MHz}$, $\left.\mathrm{CDCl}_{3}\right) \delta 7.30-7.14(\mathrm{~m}, 5 \mathrm{H}), 6.93(\mathrm{~d}, J=1.7 \mathrm{~Hz}, 1 \mathrm{H}), 6.86-6.78(\mathrm{~m}, 2 \mathrm{H}), 5.80-5.65(\mathrm{~m}, 1 \mathrm{H}), 5.08-5.01$ (m, 2H), $3.88(\mathrm{~s}, 3 \mathrm{H}), 3.86(\mathrm{~s}, 3 \mathrm{H}), 3.68-3.50(\mathrm{~m}, 3 \mathrm{H}), 2.42-2.35(\mathrm{~m}, 2 \mathrm{H}), 1.75(\mathrm{br} \mathrm{s}, 1 \mathrm{H}) ;{ }^{13} \mathrm{C}$ NMR $(125$ $\left.\mathrm{MHz}_{2} \mathrm{CDCl}_{3}\right) \delta 149.2,148.1,140.7,136.5,135.6,128.4,128.2,126.9,119.6,117.5,111.1,110.2,61.4$, 55.9 (2C), 51.5, 43.3; MS (MALDI) $\mathrm{m} / \mathrm{z}$ (relative intensity) $298\left([\mathrm{M}+1]^{+}, 63 \%\right.$ ); Anal. Calcd. for $\mathrm{C}_{19} \mathrm{H}_{23} \mathrm{NO}_{2}: \mathrm{C}, 76.73 ; \mathrm{H}, 7.80 ; \mathrm{N}, 4.71$. Found: C, 76.48; H, 7.79; N, 4.70.

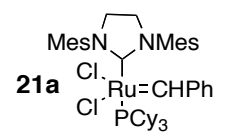

Cross metathesis (General Procedure F). To a Schlenk tube containing rac-6a or rac-6h $(0.164 \mathrm{mmol})$ and a stirring bar, was added $\mathrm{CH}_{2} \mathrm{Cl}_{2}(2.7 \mathrm{~mL})$ and the appropriate alkene (3 equiv) under argon. Second generation Grubbs catalyst 21a (5\% mol) was then added in one portion under argon. With a stream of argon applied through the sidearm of the Schlenk tube, the pink solution was heated to reflux and monitored by TLC. Upon completion of the reaction (typically $3 \mathrm{~h}$ ), the mixture was concentrated and filtered through a short plug of silica gel, eluting with EtOAc. Concentration and purification by flash chromatography gave the cross metathesis products 11-15 and 17-18. 
Friestad et al.

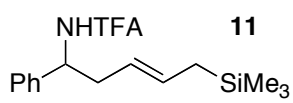

(E)-2,2,2-Trifluoro- $N$-(1-phenyl-4-trimethylsilyl-but-3-enyl)-acetamide (11). From rac-6a (40 mg, $0.164 \mathrm{mmol})$ and allyltrimethylsilane $(80 \mu \mathrm{L}, 0.492 \mathrm{mmol})$ by General Procedure F was obtained a colorless oil $(43 \mathrm{mg}, 80 \%, E / Z=2: 1)$. Further chromatography (20:1 petroleum ether-methyl $t$-butyl ether) gave 11 as a colorless oil; IR (film) 3309 , 3085, 3036, 2947, 2907, 1715, 1698, 1653, 1557, 1539, 1453, 1416, 1317, 1248, $1183 \mathrm{~cm}^{-1}$; ${ }^{1} \mathrm{H}$ NMR $\left(500 \mathrm{MHz}, \mathrm{CDCl}_{3}\right) \delta$ 7.35-7.22 (m, 5H), 6.47 (br s, 1H), 5.53 (ddd, $\left.J=16.2,8.1,8.1 \mathrm{~Hz}, 1 \mathrm{H}\right), 5.10-5.05$ (m, $1 \mathrm{H}), 4.98$ (ddd, $J=7.0,7.0,7.0 \mathrm{~Hz}, 1 \mathrm{H}), 2.60-2.55(\mathrm{~m}, 2 \mathrm{H}), 1.41(\mathrm{~d}, J=8.1 \mathrm{~Hz}, 1 \mathrm{H}),-0.06(\mathrm{~s}, 9 \mathrm{H})$; ${ }^{13} \mathrm{C} \mathrm{NMR}\left(125 \mathrm{MHz}, \mathrm{CDCl}_{3}\right) \delta 156.4\left({ }^{2} J_{\mathrm{CF}}=36.9 \mathrm{~Hz}\right), 139.6,132.1,128.9,128.0,126.4,122.0,115.9$ $\left({ }^{1} J_{\mathrm{CF}}=286.4 \mathrm{~Hz}\right), 53.8,39.1,23.1,-2.1$; MS (MALDI) $\mathrm{m} / \mathrm{z}$ (relative intensity) $330\left([\mathrm{M}+1]^{+}, 8 \%\right)$; HRMS-CI $(m / z)[\mathrm{M}+\mathrm{H}]^{+}$calcd. for $\mathrm{C}_{16} \mathrm{H}_{23} \mathrm{~F}_{3} \mathrm{NOSi}$, 330.1501; found, 330.1500.

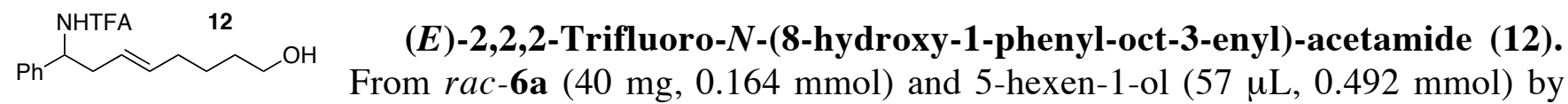
General Procedure F was obtained a colorless oil $(35 \mathrm{mg}, 68 \%, E / Z=7: 1)$. Further chromatography $(1: 1$ petroleum ether-methyl $t$-butyl ether) gave 12 as a colorless oil; IR (film) 3296, 3071, 3036, 2935, 2862, $1700,1653,1558,1537,1456,1369,1208,1185,1159,1056 \mathrm{~cm}^{-1} ;{ }^{1} \mathrm{H} \mathrm{NMR}\left(500 \mathrm{MHz}, \mathrm{CDCl}_{3}\right) \delta 7.36-$ $7.23(\mathrm{~m}, 5 \mathrm{H}), 6.52$ (br s, $1 \mathrm{H}), 5.54(\mathrm{ddd}, J=15.2,6.8,6.8 \mathrm{~Hz}, 1 \mathrm{H}), 5.31-5.26(\mathrm{~m}, 1 \mathrm{H}), 5.01(\mathrm{ddd}, J=$ 7.0, 7.0, 7.0 Hz, 1H), 3.59 (dd, $J=6.4,6.4 \mathrm{~Hz}, 2 \mathrm{H}), 2.59-2.55(\mathrm{~m}, 2 \mathrm{H}), 2.01-1.97(\mathrm{~m}, 2 \mathrm{H}), 1.50-1.38(\mathrm{~m}$, $4 \mathrm{H}), 1.21$ (br s, $1 \mathrm{H}) ;{ }^{13} \mathrm{C} \mathrm{NMR}\left(125 \mathrm{MHz}, \mathrm{CDCl}_{3}\right) \delta 156.4\left({ }^{2} J_{\mathrm{CF}}=36.9 \mathrm{~Hz}\right), 139.6,135.2,128.8,127.9$, 126.3, 124.4, $115.9\left({ }^{1} J_{\mathrm{CF}}=286.6 \mathrm{~Hz}\right), 62.5,53.5,38.7,32.1,32.0,25.2$; MS (MALDI) $\mathrm{m} / z$ (relative intensity) $316\left([\mathrm{M}+1]^{+}, 23 \%\right)$; Anal. Calcd. for $\mathrm{C}_{16} \mathrm{H}_{20} \mathrm{~F}_{3} \mathrm{NO}_{2}: \mathrm{C}, 60.94 ; \mathrm{H}, 6.39 ; \mathrm{N}, 4.44$. Found: C, $60.95 ; \mathrm{H}, 6.45 ; \mathrm{N}, 4.27$.

${ }^{\text {NHTFA }}{ }^{13} \quad$ 5-Phenyl-5-(2,2,2-trifluoro-acetylamino)-pent-2-enoic acid methyl ester (13). From rac-6h (40 mg, $0.164 \mathrm{mmol})$ and methyl acrylate $(45 \mu \mathrm{L}, 0.492 \mathrm{mmol})$ by General Procedure F was obtained $13(45 \mathrm{mg}, 86 \%, E / Z=4.5: 1)$ as a colorless solid; $\mathrm{mp} 87-88{ }^{\circ} \mathrm{C}$; IR (film) 3307, 3090, 3038, 2950, 1701, 1651, 1556, 1542, 1438, 1281, 1204, 1178, $1036 \mathrm{~cm}^{-1}$; ${ }^{1} \mathrm{H}$ NMR $\left(500 \mathrm{MHz}, \mathrm{CDCl}_{3}\right) \delta 7.38-7.25(\mathrm{~m}, 5 \mathrm{H}), 6.76(\mathrm{ddd}, J=16.0,7.5,7.5 \mathrm{~Hz}, 1 \mathrm{H}), 6.69(\mathrm{br} \mathrm{d}, J=6.0 \mathrm{~Hz}$, $1 \mathrm{H}), 5.86$ (dddd, $J=16.0,1.5,1.5,1.5 \mathrm{~Hz}, 1 \mathrm{H}), 5.12(\mathrm{ddd}, J=7.5,7.5,7.5 \mathrm{~Hz}, 1 \mathrm{H}), 3.68(\mathrm{~s}, 3 \mathrm{H}), 2.85-$ $2.76(\mathrm{~m}, 2 \mathrm{H}) ;{ }^{13} \mathrm{C}$ NMR $\left(125 \mathrm{MHz}, \mathrm{CDCl}_{3}\right) \delta 208.9,166.2,156.6\left({ }^{2} J_{\mathrm{CF}}=36.9 \mathrm{~Hz}\right), 142.6,138.6,129.3$, 128.6, 126.5, 124.7, $115.8\left({ }^{1} J_{\mathrm{CF}}=286.5 \mathrm{~Hz}\right), 53.2$, 51.6, 37.8; Anal. Calcd. for $\mathrm{C}_{14} \mathrm{H}_{14} \mathrm{~F}_{3} \mathrm{NO}_{3}: \mathrm{C}, 55.82$; H, 4.68; N, 4.65. Found: C, 55.98; H, 4.62; N, 4.72 .

NHTFA 14 O (E)-2,2,2-Trifluoro- $N$-(7-oxo-1-phenyl-3-oct-enyl)-acetamide (14). From rac6a (40 mg, $0.164 \mathrm{mmol})$ and 5 -hexen-2-one $(59 \mu \mathrm{L}, 0.492 \mathrm{mmol}$ ) by General Procedure $\mathrm{F}$ was obtained a colorless oil $(37 \mathrm{mg}, 72 \%, E / Z=4.5: 1)$. Further chromatography $(1: 1$ hexane-diethyl ether) gave $(E)-\mathbf{1 4}$ as a colorless solid; $(E)-14$ : mp 49-50 ${ }^{\circ} \mathrm{C}$; IR (film) 3348, 3080, 3028, 2925, 2852, 1715, 1698, 1648, 1542, 1439, 1364, 1222, 1181, $1156 \mathrm{~cm}^{-1} ;{ }^{1} \mathrm{H} \mathrm{NMR}\left(500 \mathrm{MHz}, \mathrm{CDCl}_{3}\right) \delta$ 7.35-7.23 (m, 5H), $6.69(\mathrm{~m}, 1 \mathrm{H}), 5.51(\mathrm{ddd}, J=15.0,6.7,6.7 \mathrm{~Hz}, 1 \mathrm{H}), 5.32-5.26(\mathrm{~m}, 1 \mathrm{H}), 5.03(\mathrm{dd}, J=$ 7.6, $6.8 \mathrm{~Hz}, 1 \mathrm{H}), 2.55-2.52(\mathrm{~m}, 2 \mathrm{H}), 2.49-2.38(\mathrm{~m}, 2 \mathrm{H}), 2.25-2.21(\mathrm{~m}, 2 \mathrm{H}), 2.08(\mathrm{~s}, 3 \mathrm{H}) ;{ }^{13} \mathrm{C}$ NMR $(125$ $\left.\mathrm{MHz}, \mathrm{CDCl}_{3}\right) \delta 207.9,156.4\left({ }^{2} J_{\mathrm{CF}}=36.9 \mathrm{~Hz}\right), 139.5,133.6,128.8,127.9,126.3,125.1,115.9\left({ }^{1} J_{\mathrm{CF}}=\right.$ 286.4 Hz), 53.3, 42.7, 38.6, 29.8, 26.5; MS (MALDI) $\mathrm{m} / \mathrm{z}$ (relative intensity) 314 ([M+1] $]^{+}, 31 \%$ ); Anal. Calcd. for $\mathrm{C}_{16} \mathrm{H}_{18} \mathrm{~F}_{3} \mathrm{NO}_{2}$ : C, 61.33; H, 5.79; N, 4.47. Found: C, 61.54; H, 5.88; N, 4.36. (Z)-14: Colorless oil; IR (film) 3308, 3080, 3007, 2919, 1715, 1701, 1651, 1557, 1540, 1455, 1364, 1204, 1204, 1180, $1157 \mathrm{~cm}^{-1} ;{ }^{1} \mathrm{H}$ NMR $\left(500 \mathrm{MHz}, \mathrm{CDCl}_{3}\right) \delta$ 7.34-7.27 (m, 6H), 5.41-5.28 (m, 2H), 5.03 (ddd, J= 8.7, 5.8, $5.8 \mathrm{~Hz}, 1 \mathrm{H}), 2.96-2.89(\mathrm{~m}, 1 \mathrm{H}), 2.63-2.46(\mathrm{~m}, 4 \mathrm{H}), 2.12(\mathrm{~s}, 3 \mathrm{H}), 2.07-2.02(\mathrm{~m}, 1 \mathrm{H}) ;{ }^{13} \mathrm{C}$ NMR $(125$ $\left.\mathrm{MHz}, \mathrm{CDCl}_{3}\right) \delta 209.2,156.6\left({ }^{2} J_{\mathrm{CF}}=36.9 \mathrm{~Hz}\right), 140.7,131.5,128.8,127.8,126.3,125.8,115.7\left({ }^{1} J_{\mathrm{CF}}=\right.$ $286.4 \mathrm{~Hz}), 53.9,42.5,33.8,30.0,21.2$. 
$\overbrace{}^{\mathrm{NHTFA}}$

(E)-2,2,2-Trifluoro- $N$-(5-hydroxy-1-phenyl-3-oct-enyl)-acetamide (15). From rac-6a $(40 \mathrm{mg}, 0.164 \mathrm{mmol})$ and $r a c-1$-hepten-3-ol $(\mathbf{1 6}, 60 \mu \mathrm{L}, 0.492 \mathrm{mmol})$ by General Procedure F was obtained diastereomeric mixture $(E)-\mathbf{1 5}\left(32 \mathrm{mg}, 61 \%\right.$, dr $3: 2$ by $\left.{ }^{13} \mathrm{C} \mathrm{NMR}\right)$ as a colorless oil. IR (film) 3334, 3080, 3033, 2961, 2925, 2873, 1694, 1542, 1456, 1248, 1177, 1075, 1023, $1000 \mathrm{~cm}^{-1} ;{ }^{1} \mathrm{H}$ NMR $\left(500 \mathrm{MHz}, \mathrm{CDCl}_{3}\right) \delta$ 7.40-7.24 (m, 5H), $6.46(\mathrm{br} \mathrm{s}, 1 \mathrm{H}), 5.61-5.46(\mathrm{~m}, 2 \mathrm{H}), 5.05$ (ddd, $J=7.5,7.5,7.5 \mathrm{~Hz}, 1 \mathrm{H}), 4.03(\mathrm{dd}, J=6.0,6.0 \mathrm{~Hz}, 1 \mathrm{H}), 2.67-2.58(\mathrm{~m}, 2 \mathrm{H}), 1.50-1.15(\mathrm{~m}, 5 \mathrm{H})$, $0.89(\mathrm{t}, J=7.0 \mathrm{~Hz}, 3 \mathrm{H}) ;{ }^{13} \mathrm{C}$ NMR $\left(125 \mathrm{MHz}, \mathrm{CDCl}_{3}\right) \delta 156.5\left({ }^{2} J_{\mathrm{CF}}=36.9 \mathrm{~Hz}\right), 139.4$ (major), 139.3 (minor), 137.9, 128.9, 128.1, 126.4, 125.1, 125.0, $115.8\left({ }^{1} J_{\mathrm{CF}}=286.6 \mathrm{~Hz}\right.$ ), 72.15 (major), 72.10 (minor), 53.5, 39.28 (major), 39.25 (minor), 38.34 (major), 38.31 (minor), 18.5, 13.8; MS (EI) $\mathrm{m} / \mathrm{z}$ (relative intensity) $297\left(\left[\mathrm{M}-\mathrm{H}_{2} \mathrm{O}\right]^{+}, 9 \%\right)$.

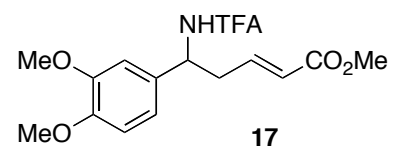

(E)-5-(3,4-Dimethoxy-phenyl)-5-(2,2,2-trifluoro-acetylamino)-pent-2enoic acid methyl ester (17). From rac-6h $(50 \mathrm{mg}, 0.164 \mathrm{mmol})$ and methyl acrylate $(45 \mu \mathrm{L}, 0.492 \mathrm{mmol})$ by General Procedure $\mathrm{F}$ was obtained a colorless oil (58 mg, 98\%, $E / Z=5: 1)$. Further chromatography gave $(E)-\mathbf{1 7}$ and $(Z)-\mathbf{1 7}$ as colorless oils. (E)-17: IR (film) 3302, 3080, 3007, 2950, 2842, 1718, 1699, 1651, 1555, 1542, 1519, 1460, 1436, 1426, 1263, 1209, 1178, 1160, $1026 \mathrm{~cm}^{-1} ;{ }^{1} \mathrm{H}$ NMR $\left(500 \mathrm{MHz}, \mathrm{CDCl}_{3}\right) \delta 6.88(\mathrm{br} \mathrm{d}, J=6.0 \mathrm{~Hz}, 1 \mathrm{H}), 6.81(\mathrm{~s}, 2 \mathrm{H})$, 6.78-6.71 (m, 2H), $5.84(\mathrm{~d}, J=15.5 \mathrm{~Hz}, 1 \mathrm{H}), 5.03(\mathrm{dd}, J=15.0,7.5 \mathrm{~Hz}, 1 \mathrm{H}), 3.82(\mathrm{~s}, 3 \mathrm{H}), 3.81(\mathrm{~s}, 3 \mathrm{H})$, $3.66(\mathrm{~s}, 3 \mathrm{H}), 2.85-2.71(\mathrm{~m}, 2 \mathrm{H}) ;{ }^{13} \mathrm{C}$ NMR $\left(125 \mathrm{MHz}, \mathrm{CDCl}_{3}\right) \delta 166.3,156.5\left({ }^{2} J_{\mathrm{CF}}=36.8 \mathrm{~Hz}\right), 149.4$, 149.2, 142.9, 131.1, 124.3, 118.7, $115.8\left({ }^{1} J_{\mathrm{CF}}=286.5 \mathrm{~Hz}\right), 111.6,110.3,55.94,55.90,52.97,51.5,37.5$; MS (MALDI) $\mathrm{m} / \mathrm{z}$ (relative intensity) $362\left([\mathrm{M}+1]^{+}, 7 \%\right)$; HRMS-CI $(\mathrm{m} / z)[\mathrm{M}+\mathrm{Na}]^{+}$calcd. for $\mathrm{C}_{16} \mathrm{H}_{18} \mathrm{~F}_{3} \mathrm{NO}_{5} \mathrm{Na}$, 384.1035; found, 384.1032; Anal. Calcd. for $\mathrm{C}_{16} \mathrm{H}_{18} \mathrm{~F}_{3} \mathrm{NO}_{5}$ : C, 53.19; H, 5.02; N, 3.88 . Found: C, 53.41; H, 4.94; N, 3.85. (Z)-17: Colorless oil; IR (film) 3306, 3080, 3007, 2945, 2832, 1717, 1702, 1650, 1557, 1540, 1519, 1454, 1440, 1258, 1209, 1178, 1163, $1026 \mathrm{~cm}^{-1} ;{ }^{1} \mathrm{H}$ NMR $(500 \mathrm{MHz}$, $\left.\mathrm{CDCl}_{3}\right) \delta 7.85(\mathrm{br} \mathrm{d}, J=6.5 \mathrm{~Hz}, 1 \mathrm{H}), 6.90-6.82(\mathrm{~m}, 3 \mathrm{H}), 6.28-6.22(\mathrm{~m}, 1 \mathrm{H}), 6.00(\mathrm{~d}, J=11.0 \mathrm{~Hz}, 1 \mathrm{H})$, 5.02-4.95 (m, 1H), $3.87(\mathrm{~s}, 3 \mathrm{H}), 3.85(\mathrm{~s}, 3 \mathrm{H}), 3.76(\mathrm{~s}, 3 \mathrm{H}), 3.45-3.37(\mathrm{~m}, 1 \mathrm{H}), 2.76-2.68(\mathrm{~m}, 1 \mathrm{H}) ;{ }^{13} \mathrm{C}$ $\operatorname{NMR}\left(125 \mathrm{MHz}, \mathrm{CDCl}_{3}\right) \delta 167.9,156.8\left({ }^{2} J_{\mathrm{CF}}=36.8 \mathrm{~Hz}\right), 149.3,148.9,143.9,132.7,123.1,118.4,115.8$ $\left({ }^{1} J_{\mathrm{CF}}=286.4 \mathrm{~Hz}\right), 111.5,109.8,55.95,55.93,53.3,51.7,34.9$.

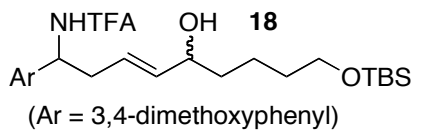

$N$-[9-(tert-Butyl-dimethyl-silanyloxy)-1-(3,4-dimethoxy-phenyl)-5-

hydroxy-3-enyl]- 2,2,2-trifluoro-acetamide (18). From rac-6h (40 mg, $0.164 \mathrm{mmol})$ and 7-(tert-butyl-dimethyl-silanyloxy)-1-hepten-3-ol ${ }^{6,7}(\mathbf{1 9}, 120$ $\mathrm{mg}, 0.492 \mathrm{mmol})$ by General Procedure F was obtained $18(25 \mathrm{mg}, 30 \%, E / Z=>20: 1)$ as a colorless oil; IR (film) 3347, 3250, 3080, 2933, 2859, 1697, 1517, 1458, 1256, 1153, $1022 \mathrm{~cm}^{-1}$; ${ }^{1} \mathrm{H}$ NMR $(500 \mathrm{MHz}$, $\left.\mathrm{CDCl}_{3}\right) \delta$ 6.85-6.79 (m, 2H), $6.76(\mathrm{~s}, 1 \mathrm{H}), 6.40(\mathrm{br} \mathrm{d}, J=8.5 \mathrm{~Hz}, 1 \mathrm{H}), 5.62-5.48(\mathrm{~m}, 2 \mathrm{H}), 4.99$ (ddd, $J=$ 7.5, 7.5, 7.5 Hz, 1H), $4.03(\mathrm{br} \mathrm{s}, 1 \mathrm{H}), 3.86(\mathrm{~s}, 3 \mathrm{H}), 3.85(\mathrm{~s}, 3 \mathrm{H}), 3.61-3.55(\mathrm{~m}, 4 \mathrm{H}), 3.58(\mathrm{t}, J=6.5 \mathrm{~Hz}$, $2 \mathrm{H}), 2.61(\mathrm{t}, J=6.5 \mathrm{~Hz}, 2 \mathrm{H}), 1.56-1.43(\mathrm{~m}, 7 \mathrm{H}), 0.87(\mathrm{~s}, 9 \mathrm{H}), 0.02(\mathrm{~s}, 6 \mathrm{H}) ;{ }^{13} \mathrm{C} \mathrm{NMR}\left(125 \mathrm{MHz}, \mathrm{CDCl}_{3}\right)$ $\delta 156.3\left({ }^{2} J_{\mathrm{CF}}=37.6 \mathrm{~Hz}\right), 149.3,137.7,133.7,131.9,125.1,118.5,115.8\left({ }^{1} J_{\mathrm{CF}}=287.5 \mathrm{~Hz}\right), 111.5,110.2$, 72.3, 63.0, 56.05, 56.00, 53.4, 38.2, 36.9, 32.6, 25.9, 21.6, 18.2; HRMS-EI $(\mathrm{m} / \mathrm{z})[\mathrm{M}]^{+}$calcd. for $\mathrm{C}_{25} \mathrm{H}_{40} \mathrm{~F}_{3} \mathrm{NO}_{5} \mathrm{Si}, 519.2639$; found, 519.2636.

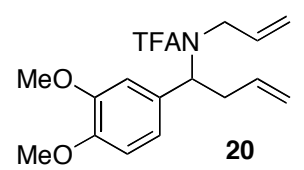

(S)-2,2,2-Trifluoro- $N$-allyl- $N$-(1-(3,4-dimethoxyphenyl)-1-butenyl)acetamide (20). To a solution of trifluoroacetamide rac-6h $(53 \mathrm{mg}, 0.175 \mathrm{mmol})$ in THF (1 mL) was added lithium hexamethyldisilazide $(0.18 \mathrm{~mL}, 1 \mathrm{M}$ in THF, $0.87 \mathrm{mmol})$ and allyl iodide $(0.02 \mathrm{~mL}, 0.2 \mathrm{mmol})$. Monitoring by TLC showed no reaction. Additional allyl iodide $(0.2 \mathrm{~mL}, 2 \mathrm{mmol})$ and HMPA $(0.1 \mathrm{~mL})$ were added at ambient temperature, and the mixture was stirred in the dark for $16 \mathrm{~h}$. Concentration and flash chromatography gave $N$-allyl product 20 (35 $\mathrm{mg}, 58 \%)$ as a colorless liquid followed by unreacted $\mathbf{6 h}(17 \mathrm{mg}, 32 \%)$. Complicated NMR spectra 
were observed for $\mathbf{2 0}$ as a result of hindered rotation. IR (film) 3056, 2982, 2939, 2834, 2300, 1686, 1604, 1521, 1477, 1265, 1208, $1152 \mathrm{~cm}^{-1} ;{ }^{1} \mathrm{H}$ NMR (300 MHz, $\left.\mathrm{CDCl}_{3}\right) \delta$ 6.92-6.80 (m, 3H), 5.79-5.66 $(\mathrm{m}, 2.5 \mathrm{H}), 5.60-5.48(\mathrm{~m}, 4.5 \mathrm{H}), 3.93-3.67(\mathrm{~m}, 8 \mathrm{H}), 2.93-2.61(\mathrm{~m}, 2 \mathrm{H}) ;{ }^{13} \mathrm{C} \mathrm{NMR}\left(75 \mathrm{MHz}, \mathrm{CDCl}_{3}\right) \delta$ $157.4\left({ }^{2} J_{\mathrm{CF}}=35.3 \mathrm{~Hz}\right), 149.1,134.2,134.0,133.1,132.2,129.8,128.8,120.7,120.1,118.8,118.0$, $117.8,116.6\left({ }^{1} J_{\mathrm{CF}}=287 \mathrm{~Hz}\right), 112.0,111.5,110.8,110.7,59.5,59.4,55.9,55.8,47.3,46.1,35.9,35.2$; MS (EI) $\mathrm{m} / z$ (relative intensity) $343.01\left([\mathrm{M}]^{+}\right.$, 14\%); Anal. Calcd. for $\mathrm{C}_{17} \mathrm{H}_{20} \mathrm{~F}_{3} \mathrm{NO}_{3}$ : C, 59.47; $\mathrm{H}, 5.87$; N, 4.08. Found: C, 59.49; H, 5.90; N, 4.03.

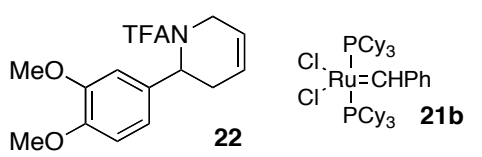

(S)-2-(3,4-Dimethoxyphenyl)- $N$-2,2,2-trifluoroacetyl-1,2,3,6tetrahydropyridine (22). To a solution of $N$-allyltrifluoroacetamide $\mathbf{2 0}$ (25 mg, $0.072 \mathrm{mmol})$ in $\mathrm{CH}_{2} \mathrm{Cl}_{2}(36 \mathrm{~mL})$ was added Grubbs catalyst $\mathbf{2 1 b}$ (5.9 $\mathrm{mg}, 0.0072 \mathrm{mmol})$. After $16 \mathrm{~h}$ at ambient temperature, concentration and flash chromatography gave $22(21 \mathrm{mg}, 92.5 \%)$ as a colorless liquid. Complicated NMR spectra were observed for $\mathbf{2 2}$ as a result of hindered rotation (two rotamers, ratio 3:2). IR (Film) 2929, 2851, 1687, 1518, 1451, 1257, 1188, 1143, $1027 \mathrm{~cm}^{-1}$; ${ }^{1} \mathrm{H}$ NMR $\left(300 \mathrm{MHz}, \mathrm{CDCl}_{3}\right) \delta$ 6.90-6.78 (m, 3H), 6.06$5.90(\mathrm{~m}, 1 \mathrm{H}), 5.98(\mathrm{~d}, \mathrm{~J}=6.6 \mathrm{~Hz}, 0.6 \mathrm{H}), 5.66-5.61(\mathrm{~m}, 1 \mathrm{H}), 5.30(\mathrm{~d}, J=5.7 \mathrm{~Hz}, 0.4 \mathrm{H}), 4.61(\mathrm{br} \mathrm{d}, J=$ $18.1 \mathrm{~Hz}, 0.4 \mathrm{H}), 4.14$ (br d, $J=17.8 \mathrm{~Hz}, 0.6 \mathrm{H}), 3.86$ (s, 3H), $3.83(\mathrm{~s}, 3 \mathrm{H}), 3.56-3.49$ (m, 0.6H), 3.24 (br $\mathrm{d}, J=18.9 \mathrm{~Hz}, 0.4 \mathrm{H}), 2.78-2.65(\mathrm{~m}, 2 \mathrm{H}) ;{ }^{13} \mathrm{C} \mathrm{NMR}\left(75 \mathrm{MHz}, \mathrm{CDCl}_{3}\right) \delta 156.2\left({ }^{2} J_{\mathrm{CF}}=35.3 \mathrm{~Hz}\right), 155.7(J$ $=35.5 \mathrm{~Hz}), 149.1,148.8,130.6,129.8,124.7,123.9,123.4,122.7,119.5,119.2,116.7\left({ }^{1} J_{\mathrm{CF}}=286 \mathrm{~Hz}\right)$, 111.0, 110.9, 110.1, 56.0, 55.9, 53.5, 50.1, 40.9, 39.7, 28.2, 26.9; MS (EI) $\mathrm{m} / \mathrm{z}$ (relative intensity) 315.2 $\left([\mathrm{M}]^{+}, 42 \%\right)$; Anal. Calcd. for $\mathrm{C}_{15} \mathrm{H}_{16} \mathrm{~F}_{3} \mathrm{NO}_{3}$ : C, 57.14; H, 5.12; N, 4.44. Found: C, 57.32; H, 5.09; N, 4.40.

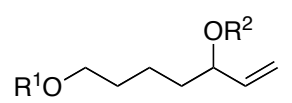

i $\left(R^{1}=T B S, R^{2}=H\right)$ ii $\left(\mathrm{R}^{1}=\mathrm{TBS}, \mathrm{R}^{2}=\mathrm{CO}_{2} \mathrm{Me}\right)$ iii $\left(\mathrm{R}^{1}=\mathrm{H}, \mathrm{R}^{2}=\mathrm{CO}_{2} \mathrm{Me}\right)$ $23\left(R^{1}=\mathrm{Ts}, \mathrm{R}^{2}=\mathrm{CO}_{2} \mathrm{Me}\right)$
Toluene-4-sulfonic acid 5-methoxycarbonyloxy-hept-6-enyl ester (23). To a solution of $\mathbf{i}^{6,7}(1.61 \mathrm{~g}, 6.59 \mathrm{mmol})$, pyridine $(6 \mathrm{~mL})$ and DMAP (ca. $\left.5 \mathrm{mg}\right)$ in $\mathrm{CH}_{2} \mathrm{Cl}_{2}(20 \mathrm{~mL})$ at $0{ }^{\circ} \mathrm{C}$ was added methyl chloroformate $(0.8 \mathrm{~mL}, 10 \mathrm{mmol})$. The mixture was allowed to warm to ambient temperature over ca. $16 \mathrm{~h}$, then partitioned between saturated $\mathrm{NH}_{4} \mathrm{Cl}$ solution and $\mathrm{Et}_{2} \mathrm{O}$. The organic phase was washed with $2 \mathrm{M} \mathrm{HCl}$, water, and brine, then dried $\left(\mathrm{MgSO}_{4}\right)$. Concentration and flash chromatography (hexane/ $\left.\mathrm{Et}_{2} \mathrm{O} 2: 1\right)$ gave the carbonate ii $(1.52 \mathrm{~g}, 72 \%)$ as a pale yellow oil. To a solution of ii in THF $(11 \mathrm{~mL})$ was added TBAF $(6.5 \mathrm{~mL}, 1.0 \mathrm{M}$ in THF, $6.5 \mathrm{mmol})$. After ca. $16 \mathrm{~h}$, concentration and flash chromatography (hexane/ $\left.\mathrm{Et}_{2} \mathrm{O} 1: 2\right)$ gave the primary alcohol iii $(0.79 \mathrm{~g}, 84 \%)$ as a pale yellow oil. To a solution of iii (445 mg, $2.36 \mathrm{mmol})$, DMAP (ca. $5 \mathrm{mg}$ ) and $\mathrm{Et}_{3} \mathrm{~N}(0.60 \mathrm{~mL}, 4.3$ mmol) in $\mathrm{CH}_{2} \mathrm{Cl}_{2}$ at $0{ }^{\circ} \mathrm{C}$ was added a solution of $p$-toluenesulfonyl chloride $(680 \mathrm{mg}, 3.53 \mathrm{mmol})$ in $\mathrm{CH}_{2} \mathrm{Cl}_{2}(18 \mathrm{~mL})$. The clear solution was allowed to warm to ambient temperature in $22 \mathrm{~h}$, then partitioned between water and $\mathrm{CH}_{2} \mathrm{Cl}_{2}$. The organic phase was washed with saturated $\mathrm{NaHCO}_{3}$ solution, $1 \mathrm{M} \mathrm{HCl}$, and brine, then dried $\left(\mathrm{MgSO}_{4}\right)$. Concentration and flash chromatography furnished tosylate 23 (686 mg, 85\%) as a colorless oil. IR (film): 2956, 2870, 1747, 1598, 1443, 1359, 1266, 1176, $1097 \mathrm{~cm}^{-}$ ${ }^{1} ;{ }^{1} \mathrm{H}$ NMR $\left(500 \mathrm{MHz}, \mathrm{CDCl}_{3}\right) \delta 7.74(\mathrm{~d}, J=8.0 \mathrm{~Hz}, 2 \mathrm{H}), 7.31(\mathrm{~d}, J=8.0 \mathrm{~Hz}, 2 \mathrm{H}), 5.71(\mathrm{ddd}, J=17.5$, $10.5,7.0 \mathrm{~Hz}, 1 \mathrm{H}), 5.23$ (ddd, $J=17.5,1.0,1.0 \mathrm{~Hz}, 1 \mathrm{H}), 5.16$ (ddd, $J=10.0,1.0,1.0 \mathrm{~Hz}, 1 \mathrm{H}$ ), 4.96 (ddd, $J=7.0,7.0,7.0 \mathrm{~Hz}, 1 \mathrm{H}), 3.98(\mathrm{dd}, J=6.0,6.0 \mathrm{~Hz}, 2 \mathrm{H}), 3.73(\mathrm{~s}, 3 \mathrm{H}), 2.41(\mathrm{~s}, 3 \mathrm{H}), 1.73-1.26(\mathrm{~m}, 6 \mathrm{H})$; ${ }^{13} \mathrm{C} \mathrm{NMR}\left(125 \mathrm{MHz}, \mathrm{CDCl}_{3}\right) \delta 155.2,144.7,135.6,133.3,129.9,127.9,117.7,78.6,70.1,54.6,33.5$, 28.6, 21.6, 21.0; Anal. Calcd. for $\mathrm{C}_{16} \mathrm{H}_{22} \mathrm{O}_{6} \mathrm{~S}$ : C, 56.12; H, 6.48. Found: C, 56.33; H, 6.50.

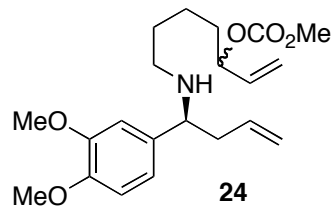

7-((S)-1-(3,4-Dimethoxyphenyl)but-3-enylamino)hept-1-en-3-yl methyl carbonate (24). To a solution of amine $7(187 \mathrm{mg}, 0.903 \mathrm{mmol})$, tosylate 23 (257 $\mathrm{mg}, 0.750 \mathrm{mmol})$ and $\mathrm{Et}_{3} \mathrm{~N}(0.11 \mathrm{~mL}, 0.825 \mathrm{mmol})$ in acetonitrile $(9.0 \mathrm{~mL})$, was added sodium iodide $(169 \mathrm{mg}, 1.12 \mathrm{mmol})$ in one portion under argon. The 
resulting white suspension was heated at reflux for $4 \mathrm{~d}$. Basic brine $(2 \mathrm{M} \mathrm{NaOH}$ saturated with $\mathrm{NaCl}$ ) was added to the cooled reaction mixture and the mixture was extracted with $\mathrm{CH}_{2} \mathrm{Cl}_{2}$ and dried over $\mathrm{MgSO}_{4}$. Concentration and flash chromatography (1:1 Hexane-EtOAc to $30: 1 \mathrm{CH}_{2} \mathrm{Cl}_{2}-\mathrm{MeOH}$ to $5: 1$ $\left.\mathrm{CH}_{2} \mathrm{Cl}_{2}-\mathrm{MeOH}\right)$ afforded unreacted amine $7(48 \mathrm{mg})$ and $24(183 \mathrm{mg}, 65 \%$, dr 1:1) as a pale yellow oil; IR (film) 3427, 3243, 3049, 2985, 2929, 1724, 1652, 1537, 1483, 1455, 1385, 1355, 1334, 1150, $907 \mathrm{~cm}^{-}$ ${ }^{1} ;{ }^{1} \mathrm{H}$ NMR $\left(500 \mathrm{MHz}, \mathrm{CDCl}_{3}\right) \delta 6.86(\mathrm{~s}, 1 \mathrm{H}), 6.80-6.77(\mathrm{~m}, 2 \mathrm{H}), 5.77-5.63(\mathrm{~m}, 2 \mathrm{H}), 5.23(\mathrm{~d}, J=17.0$ $\mathrm{Hz}, 1 \mathrm{H}), 5.15(\mathrm{~d}, J=10.5 \mathrm{~Hz}, 1 \mathrm{H}), 5.09-4.96(\mathrm{~m}, 2 \mathrm{H}), 3.85$ (s, 3H), 3.83 (s, 3H), 3.72 (s, 3H), 3.53 (dd, $J=7.5,6.0 \mathrm{~Hz}, 1 \mathrm{H}), 2.44-2.27(\mathrm{~m}, 4 \mathrm{H}), 1.68-1.22(\mathrm{~m}, 8 \mathrm{H}) ;{ }^{13} \mathrm{C} \mathrm{NMR}\left(125 \mathrm{MHz}, \mathrm{CDCl}_{3}\right) \delta 155.3,149.1$, 148.0, 136.8, 136.0, 135.6, 119.4, 117.4 (2C), 111.0, 110.0, 79.0, 78.97, 62.4, 55.9, 54.6, 47.4, 43.2, 34.1, 29.9, 22.7; Anal. Calcd. for $\mathrm{C}_{21} \mathrm{H}_{31} \mathrm{NO}_{5}$ : C, 66.82; H, 8.28; N, 3.71. Found: C, 66.82; H, 8.26; N, 3.91 .

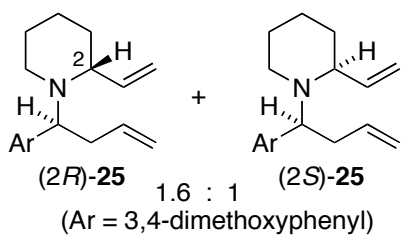

\section{(R)-1-((S)-1-(3,4-Dimethoxyphenyl)but-3-enyl)-2-vinylpiperidine (2R- $25)$ and $(S)$-1-((S)-1-(3,4-dimethoxyphenyl)but-3-enyl)-2-vinylpiperidine (2S-25). To a solution of amine $24(\mathrm{dr} 1: 1,84.0 \mathrm{mg}, 0.22 \mathrm{mmol})$, triphenylphosphine $(35 \mathrm{mg}, 0.13 \mathrm{mmol})$ and allylpalladium chloride dimer (13 $\mathrm{mg}, 0.036 \mathrm{mmol})$, was added $\mathrm{Et}_{3} \mathrm{~N}(80 \mu \mathrm{L}, 0.57 \mathrm{mmol})$. The resulting solution} was stirred at ambient temperature for $3 \mathrm{~d}$, then concentrated. The residue was taken up by 3:1 hexaneEtOAc and filtered, concentrated and purified by flash chromatography (5:1 to 1:1 Hexane-EtOAc) to give $25(60 \mathrm{mg}, 89 \%,(2 R):(2 S)=1.6: 1)$ as a pale yellow oil. The mixture was used in the next step without separation. Pure diastereomers were obtained by further flash chromatography. (2R)-25: pale yellow oil; $[\alpha]_{\mathrm{D}}^{22}+1.2^{\circ}\left(c 0.6, \mathrm{CHCl}_{3}\right)$; IR (film) 3074, 2931, 2851, 2795, 1639, 1590, 1514, 1463, 1416, 1322, 1255, 1235, 1145, $1030 \mathrm{~cm}^{-1} ;{ }^{1} \mathrm{H}$ NMR (500 MHz, $\left.\mathrm{CDCl}_{3}\right) \delta 6.95(\mathrm{~s}, 1 \mathrm{H}), 6.89-6.75(\mathrm{~m}, 2 \mathrm{H}), 5.93$ (ddd, $J=18.0,9.5,9.5 \mathrm{~Hz}, 1 \mathrm{H}), 5.65$ (dddd, $J=17.0,10.0,6.5,6.5 \mathrm{~Hz}, 1 \mathrm{H}), 5.21(\mathrm{~d}, J=17.5 \mathrm{~Hz}, 1 \mathrm{H})$, $5.10(\mathrm{~d}, J=10.0 \mathrm{~Hz}, 1 \mathrm{H}), 4.98(\mathrm{dd}, J=17.0,1.5 \mathrm{~Hz}, 1 \mathrm{H}), 4.90(\mathrm{~d}, J=10.0 \mathrm{~Hz}, 1 \mathrm{H}), 3.96-3.90(\mathrm{~m}, 1 \mathrm{H})$, $3.86(\mathrm{~s}, 3 \mathrm{H}), 3.84(\mathrm{~s}, 3 \mathrm{H}), 3.19(\mathrm{dd}, J=6.5,6.5 \mathrm{~Hz}, 1 \mathrm{H}), 2.63-2.41(\mathrm{~m}, 3 \mathrm{H}), 2.16(\mathrm{dd}, J=10.0,10.0 \mathrm{~Hz}$, $1 \mathrm{H}), 1.80-1.15(\mathrm{~m}, 6 \mathrm{H}) ;{ }^{13} \mathrm{C}$ NMR $\left(125 \mathrm{MHz}, \mathrm{CDCl}_{3}\right) \delta 148.6,147.7,141.6,137.0,135.2,120.7,115.9$, $115.4,112.6,110.4,62.8,62.7,55.92,55.87,45.2,34.0,30.4,26.2,23.7$; MS (APCI) $\mathrm{m} / z$ (relative intensity) $302\left([\mathrm{M}+1]^{+}\right.$, 48\%); Anal. Calcd. for $\mathrm{C}_{19} \mathrm{H}_{27} \mathrm{NO}_{2}: \mathrm{C}, 75.71 ; \mathrm{H}, 9.03 ; \mathrm{N}, 4.65$. Found: C, 75.84; $\mathrm{H}, 9.17$; N, 4.34. (2S)-25: pale yellow oil; $[\alpha]_{\mathrm{D}}^{22}+20^{\circ}$ (c $0.15, \mathrm{CHCl}_{3}$ ); IR (film) 3073, 2931, 2835, 2801, 2713, 1734, 1640, 1589, 1514, 1463, 1418, 1261, 1146, $1029 \mathrm{~cm}^{-1} ;{ }^{1} \mathrm{H}$ NMR $\left(500 \mathrm{MHz}, \mathrm{CDCl}_{3}\right) \delta$ 6.85-6.70 (m, 3H), $5.88(\mathrm{ddd}, J=17.5,8.5,8.5 \mathrm{~Hz}, 1 \mathrm{H}), 5.74(\mathrm{dddd}, J=17.0,10.5,7.0,7.0 \mathrm{~Hz}, 1 \mathrm{H})$, $5.19-5.11(\mathrm{~m}, 2 \mathrm{H}), 4.98(\mathrm{dd}, J=17.0,1.5 \mathrm{~Hz}, 1 \mathrm{H}), 4.94-4.88(\mathrm{~m}, 1 \mathrm{H}), 3.98(\mathrm{dd}, J=7.5,7.5 \mathrm{~Hz}, 1 \mathrm{H})$, $3.85(\mathrm{~s}, 3 \mathrm{H}), 3.83(\mathrm{~s}, 3 \mathrm{H}), 2.93-2.84(\mathrm{~m}, 1 \mathrm{H}), 2.72-2.46(\mathrm{~m}, 2 \mathrm{H}), 1.85-1.02(\mathrm{~m}, 8 \mathrm{H}) ;{ }^{13} \mathrm{C}$ NMR $(125$ $\left.\mathrm{MHz}_{,} \mathrm{CDCl}_{3}\right) \delta 148.1,147.7,143.8,136.9,130.3,121.1,115.5,115.1,112.9,110.4,63.7,62.7,55.9$, 55.8, 45.5, 37.2, 34.8, 26.3, 24.1; MS (MALDI) $\mathrm{m} / \mathrm{z}$ (relative intensity) 302 ([M+1 $]^{+}, 27 \%$ ); HRMS-CI $(\mathrm{m} / \mathrm{z})[\mathrm{M}+\mathrm{H}]^{+}$calcd. for $\mathrm{C}_{19} \mathrm{H}_{28} \mathrm{NO}_{2}, 302.2107$; found, 302.2112 .

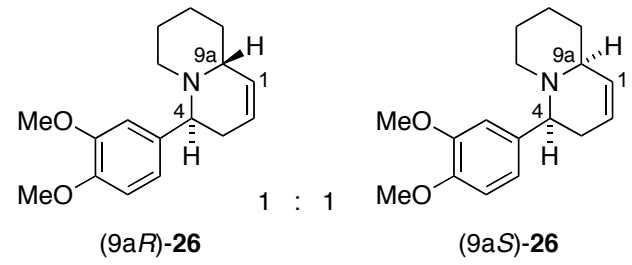

(1S,2S)-6-(3,4-Dimethoxy-phenyl)-1,3,4,6,6,7,9ahexahydro-2H-quinolizine (26). A diastereomeric mixture of 2vinylpiperidines $(2 R)-\mathbf{2 5}$ and $(2 S)-25(114 \mathrm{mg}, 0.378 \mathrm{mmol}, \mathrm{dr}=$ 1:1) and $\mathrm{TsOH} \cdot \mathrm{H}_{2} \mathrm{O}(78 \mathrm{mg}, 0.40 \mathrm{mmol})$ in $\mathrm{CH}_{2} \mathrm{Cl}_{2}(170 \mathrm{~mL})$ was stirred at ambient temperature for $3 \mathrm{~h}$. Second generation Grubbs catalyst 21 b (50 $\mathrm{mg}, 0.0588 \mathrm{mmol}$ ) was added in one portion under argon. After $2 \mathrm{~d}$ at ambient temperature, a second portion of $\mathbf{2 1 b}(20 \mathrm{mg}, 0.024 \mathrm{mmol})$ was added. After another $36 \mathrm{~h}$, the mixture was concentrated, acidified with $2 \mathrm{M} \mathrm{HCl}$, and washed with EtOAc to remove the catalyst. The acidic aqueous phase was adjusted to $\mathrm{pH} 10(2 \mathrm{M} \mathrm{NaOH})$, extracted with $\mathrm{CH}_{2} \mathrm{Cl}_{2}$, and concentrated to afford 26 as a diastereomeric mixture (dr 1:1). Flash chromatography 
(2:1 Hexane-EtOAc to 20:1 EtOAc-MeOH to 8:1 EtOAc-MeOH) furnished the two separated diastereomers (88 $\mathrm{mg}$ in total, 85\%) $(9 \mathrm{a} R)-26$ and $(9 \mathrm{a} S)$-26. $(9 \mathrm{a} R)-26$ : pale yellow oil; $[\alpha]_{\mathrm{D}}{ }^{25}-60^{\circ}(c$ $0.8, \mathrm{CHCl}_{3}$ ); IR (film) 2927, 2850, 2739, 1589, 1511, 1460, 1323, 1260, 1144, $1029 \mathrm{~cm}^{-1} ;{ }^{1} \mathrm{H}$ NMR (500 $\left.\mathrm{MHz}_{2} \mathrm{CDCl}_{3}\right) \delta 6.86(\mathrm{~s}, 1 \mathrm{H}), 6.82-6.74(\mathrm{~m}, 2 \mathrm{H}), 5.90-5.84(\mathrm{~m}, 1 \mathrm{H}), 5.57(\mathrm{dd}, J=10.0,1.5 \mathrm{~Hz}, 1 \mathrm{H}), 3.84$ $(\mathrm{s}, 3 \mathrm{H}), 3.81(\mathrm{~s}, 3 \mathrm{H}), 2.88-2.79(\mathrm{~m}, 1 \mathrm{H}), 2.72(\mathrm{~d}, J=11.5 \mathrm{~Hz}, 1 \mathrm{H}), 2.66(\mathrm{~d}, J=11.0 \mathrm{~Hz}, 1 \mathrm{H}), 2.26(\mathrm{dd}, J$ $=18.0,1.5 \mathrm{~Hz}, 1 \mathrm{H}), 2.12-1.08(\mathrm{~m}, 8 \mathrm{H}) ;{ }^{13} \mathrm{C} \mathrm{NMR}\left(125 \mathrm{MHz}, \mathrm{CDCl}_{3}\right) \delta 148.5,148.1,138.4,130.9$, $124.2,121.1,111.8,110.4,61.0,55.88,55.82,54.6,52.5,32.2,31.9,25.3,24.7$; HRMS-EI $(\mathrm{m} / z)[\mathrm{M}]^{+}$ calcd. for $\mathrm{C}_{17} \mathrm{H}_{23} \mathrm{NO}_{2}, 273.1729$; found, 273.1724. (9aS)-26: pale yellow oil; $[\alpha]_{\mathrm{D}}^{25}-58^{\circ}(c) 0.42$, $\mathrm{CHCl}_{3}$ ); IR (film) 2930, 2878, 2783 and 2728 (Bohlmann bands), 1698, 1648, 1516, 1260, 1233, 1134, $1029 \mathrm{~cm}^{-1} ;{ }^{1} \mathrm{H}$ NMR $\left(500 \mathrm{MHz}, \mathrm{CDCl}_{3}\right) \delta 6.88(\mathrm{~s}, 1 \mathrm{H}), 6.83-6.75(\mathrm{~m}, 2 \mathrm{H}), 5.76-5.69(\mathrm{~m}, 1 \mathrm{H}), 5.88(\mathrm{~d}, J$ $=9.5 \mathrm{~Hz}, 1 \mathrm{H}), 3.86(\mathrm{~s}, 3 \mathrm{H}), 3.84(\mathrm{~s}, 3 \mathrm{H}), 3.22(\mathrm{dd}, J=10.5,3.5 \mathrm{~Hz}, 1 \mathrm{H}), 2.67(\mathrm{~d}, J=11.0 \mathrm{~Hz}, 2 \mathrm{H})$, 2.45-2.38 (m, 1H), 2.25-1.15 (m, 8H); $\left.{ }^{13} \mathrm{C} \mathrm{NMR} \mathrm{(125} \mathrm{MHz,} \mathrm{CDCl}_{3}\right) \delta$ 149.3, 148.1, 136.3, 130.7, 124.6, 120.4, 110.8, 110.6, 66.1, 63.21, 56.1, 55.9, 52.5, 36.5, 32.7, 26.3, 25.1; MS (APCI) $\mathrm{m} / z$ (relative intensity) $274\left([\mathrm{M}+1]^{+}, 100 \%\right)$; HRMS-ESI $(\mathrm{m} / z)[\mathrm{M}+\mathrm{H}]^{+}$calcd. for $\mathrm{C}_{17} \mathrm{H}_{24} \mathrm{NO}_{2}, 274.1807$; found, 274.1804.

\section{Stereochemical Assignments}

\section{(a) Chemical correlation of $2 \mathrm{a}$.}

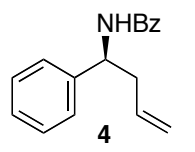

(S)-N-1-Phenyl-but-3-enyl-benzamide (4). To a solution of $\mathbf{2 a}(70 \mathrm{mg}, 0.217 \mathrm{mmol})$ in THF (2.5 mL) at $-78{ }^{\circ} \mathrm{C}$ was added $n$-BuLi $(0.18 \mathrm{~mL}, 2.5 \mathrm{M}$ in THF, $0.45 \mathrm{mmol})$. After $1 \mathrm{~h}$, benzoic anhydride (102 $\mathrm{mg}, 0.434 \mathrm{mmol})$ was added in one portion. The reaction mixture was allowed to warm to r.t. overnight, then partitioned between water and $\mathrm{CH}_{2} \mathrm{Cl}_{2}$. The organic phase was washed with brine and dried over $\mathrm{MgSO}_{4}$. Flash chromatography gave a colorless oil ( $75 \mathrm{mg}$, $81 \%)$. To a solution of this oil $(75 \mathrm{mg})$ in THF/HMPA $(20: 1,2.3 \mathrm{~mL})$ at $-78{ }^{\circ} \mathrm{C}$ was added $\mathrm{SmI}_{2}(10$ $\mathrm{mL}, 0.1 \mathrm{M}$ in THF, $1.0 \mathrm{mmol}$ ). After $10 \mathrm{~min}$, the reaction mixture was partitioned between water and $\mathrm{CH}_{2} \mathrm{Cl}_{2}$. The organic phase was washed with brine and dried over $\mathrm{MgSO}_{4}$. Flash chromatography gave 4 $(41 \mathrm{mg}, 93 \%)$ as a colorless solid. mp 98-99 ${ }^{\circ} \mathrm{C} ;[\alpha]_{\mathrm{D}}{ }^{20}-20^{\circ}\left(\mathrm{c} 0.34, \mathrm{CHCl}_{3}\right)$; spectroscopic data matched those from the literature, ${ }^{8}$ and those of an authentic sample prepared as described below. The oxazolidinone 3 was recovered (30 $\mathrm{mg}, 96 \%)$.

An authentic sample of $\mathbf{4}$ was prepared by benzoylation of the known homoallylic amine. ${ }^{9}$ Thus, to a solution of the homoallylic amine $(0.38 \mathrm{mmol})$ in $\mathrm{CH}_{2} \mathrm{Cl}_{2}(2 \mathrm{~mL})$ under ice-bath, was added pyridine $(0.38 \mathrm{mmol})$ and benzoyl chloride $(0.45 \mathrm{mmol})$. After $1 \mathrm{~h}$, water $(2 \mathrm{~mL})$ was added and the organic phase was washed with $2 \mathrm{~N} \mathrm{HCl}, 10 \% \mathrm{NaOH}$ and brine. Then it was dried over $\mathrm{MgSO}_{4}$, concentrated and purified by flash chromatography to give 4 as a colorless solid. $99-101{ }^{\circ} \mathrm{C} ;[\alpha]_{\mathrm{D}}{ }^{20}-22^{\circ}(c 0.34$, $\mathrm{CHCl}_{3}$ ); IR (film) 3334, 3064, 3030, 2916, 1634, 1592, 1491, 1304, 1073, $921 \mathrm{~cm}^{-1}$; ${ }^{1} \mathrm{H} \mathrm{NMR}(500 \mathrm{MHz}$, $\left.\mathrm{CDCl}_{3}\right) \delta 7.80-7.35(\mathrm{~m}, 10 \mathrm{H}), 6.42(\mathrm{~d}, J=6.8 \mathrm{~Hz}, 1 \mathrm{H}), 5.76(\mathrm{dddd}, J=17.1,10.1,6.7,6.7 \mathrm{~Hz}, 1 \mathrm{H}), 5.30$ (ddd, apparent q, $J=7.1 \mathrm{~Hz}, 1 \mathrm{H}), 5.17(\mathrm{ddd}, J=17.1,1.0,0.9 \mathrm{~Hz}, 1 \mathrm{H}), 5.12(\mathrm{ddd}, J=10.1,0.9,0.9 \mathrm{~Hz}$, $1 \mathrm{H}), 2.69(\mathrm{dd}$, apparent $\mathrm{t}, J=6.6 \mathrm{~Hz}, 2 \mathrm{H}) ;{ }^{13} \mathrm{C} \mathrm{NMR}\left(125 \mathrm{MHz}, \mathrm{CDCl}_{3}\right) \delta 166.8,141.8,134.7,134.2$, $131.5,128.7,128.6,127.4,127.0,126.5,118.4,52.9,40.6$; MS (EI) $m / z$ (relative intensity) $251\left([\mathrm{M}+\mathrm{H}]^{+}\right.$, $42 \%)$. 


\section{(b) Chemical correlation of $2 \mathrm{~g}$.}

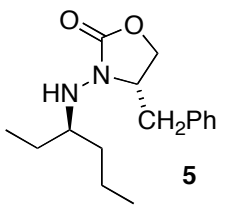

A mixture of $2 \mathrm{~g}(9 \mathrm{mg}, 0.033 \mathrm{mmol})$ and $20 \% \mathrm{Pd}(\mathrm{OH})_{2}$ on carbon $(10 \mathrm{mg})$ in methanol $(0.5 \mathrm{~mL})$ was stirred overnight under $\mathrm{H}_{2}(1 \mathrm{~atm})$. Filtration and concentration gave 5 (10 mg, 100\%) as a colorless oil; ${ }^{1} \mathrm{H}$ NMR and TLC data and HPLC retention times for both major and minor diastereomers were identical to an authentic sample produced by $n$-propyl radical addition to $\mathbf{1 g}$ (previously shown to furnish predominantly products of $\mathrm{Si}$-face attack). ${ }^{10}$

\section{(c) Assignment of cross-metathesis isomers.}

For major isomers obtained in cross-metathesis, the $E$ configuration was assigned by ${ }^{1} \mathrm{H}$ NMR spectroscopy and literature precedent according to the following analyses. (a) Large coupling constants $(15-16 \mathrm{~Hz})$ were observed for the olefinic hydrogens in the major isomers of 11-15 and 17. For 17, the major and minor isomers had $J=16$ and $11 \mathrm{~Hz}$, respectively, confirming that the $16 \mathrm{~Hz}$ coupling was associated with $(E)-\mathbf{1 7}$. (b) Furthermore, all $Z$-isomers had clearly resolved resonances for the geminal protons of the allylic $\mathrm{CH}_{2}$, while the $E$ isomers offered little or no resolution of those protons. This assignment was supported by correlation to literature data ${ }^{11}$ on related compounds $\mathbf{E}$ and $\mathbf{F}$ (shown at right); the anisotropy affecting $\mathrm{H}^{\mathrm{a}}$ and $\mathrm{H}^{\mathrm{b}}$ was associated with the $Z$ isomer. (c) Using a simplified analog $(\mathbf{G})$, conformational analysis (Chem3D) using molecular dynamics followed by energy minimization (MM2) suggested a source of the anisotropy affecting $\mathrm{H}^{\mathrm{a}}$ and $\mathrm{H}^{\mathrm{b}}$. One of these protons was clearly localized on the "inside" of the allylic system and proximal to the hydroxyl group. The extended structures of the $E$ isomers offer no obvious source of such anisotropy.

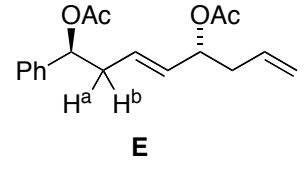

$\mathrm{H}^{\mathrm{a}}, \mathrm{H}^{\mathrm{b}}=2.48-2.65 \mathrm{ppm}$ (multiplet)

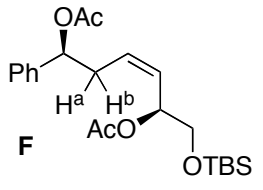

$\mathrm{H}^{\mathrm{a}}, \mathrm{H}^{\mathrm{b}}=2.68$ and $2.81 \mathrm{ppm}$ (two resolved multiplets)

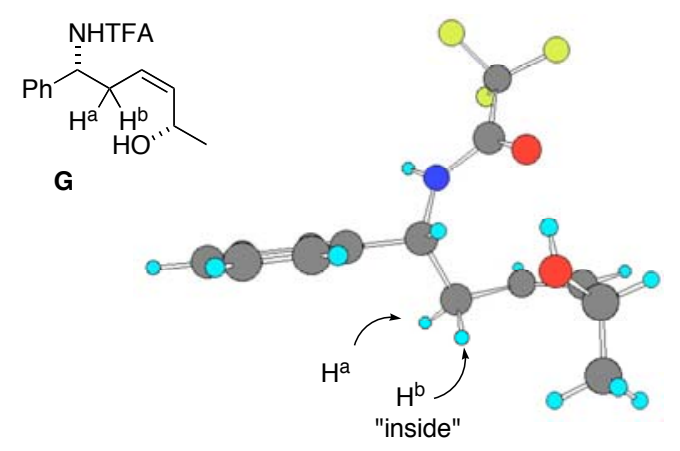

For 15 and 18, prepared using racemic allylic alcohols, four diastereomers could be envisioned. A mixture of two of the four possible diastereomers was obtained; both were assigned $E$-configuration according to the analysis above. The diastereomerism was attributed to the hydroxyl-bearing stereogenic center. The presence of small amounts $(<10 \%)$ of the $Z$ isomers in the crude product mixture was not ruled out.

\section{Effects of Chiral Ligands on Intramolecular Allylic Amination of 24.}

The procedure given for preparation of $\mathbf{2 5}$ was modified to screen the effects of several chiral ligands: From amine $24\left(6.0 \mathrm{mg}, 0.027 \mathrm{M}\right.$ in $\left.\mathrm{CH}_{2} \mathrm{Cl}_{2}, 0.016 \mathrm{mmol}\right)$, using (+)- $N, N^{\prime}$-bis(2'diphenylphosphino-benzoyl)-1,2-diaminocyclohexane $((1 R, 2 R)$-Trost ligand, $10 \mathrm{mg}, 0.014 \mathrm{mmol})$, allylpalladium chloride dimer $(1.6 \mathrm{mg}, 0.0043 \mathrm{mmol})$ and $\mathrm{Et}_{3} \mathrm{~N}(6 \mu \mathrm{L}, 0.043 \mathrm{mmol})$ by the above procedure $(2 \mathrm{~d})$, was obtained 25 as a pale yellow oil $(4.3 \mathrm{mg}, 90 \%,(2 R):(2 S)=1.05: 1)$. From amine 24 (232 mg, $0.036 \mathrm{M}$ in $\mathrm{CH}_{2} \mathrm{Cl}_{2}, 0.615 \mathrm{mmol}$ ), using (-)- $N, N^{\prime}$-bis(2'-diphenylphosphino-benzoyl)-1,2diaminocyclohexane $((1 \mathrm{~S}, 2 \mathrm{~S})$-Trost ligand, $66 \mathrm{mg}, 0.094 \mathrm{mmol})$, allylpalladium chloride dimer $(11.5$ $\mathrm{mg}, 0.031 \mathrm{mmol})$ and $\mathrm{Et}_{3} \mathrm{~N}(220 \mu \mathrm{L}, 1.57 \mathrm{mmol})$ by the above procedure $(60 \mathrm{~h})$, was obtained 25 as a 
pale yellow oil $(151 \mathrm{mg}, 81 \%,(2 R):(2 S)=1.1: 1)$. From amine $24\left(6.3 \mathrm{mg}, 0.027 \mathrm{M}\right.$ in $\mathrm{CH}_{2} \mathrm{Cl}_{2}, 0.017$ mmol), using (+)- $N, N$ '-bis(2'-diphenylphosphino-1-naphthoyl)-1,2-diaminocyclohexane ((1R,2R)-Trost ligand (naphthyl), $11 \mathrm{mg}, 0.013 \mathrm{mmol}$ ), allylpalladium chloride dimer $(1.5 \mathrm{mg}, 0.0041 \mathrm{mmol}) \mathrm{and} \mathrm{Et}_{3} \mathrm{~N}$ $(6 \mu \mathrm{L}, 0.043 \mathrm{mmol})$ by the above procedure $(3 \mathrm{~d})$, was obtained 25 as a pale yellow oil $(4.3 \mathrm{mg}, 86 \%$, $(2 R):(2 S)=1: 1)$.

\section{References and Notes}

(1) Handy, C. J.; Lam, Y.-F.; DeShong, P. J. Org. Chem. 2000, 65, 3542-3543.

(2) Friestad, G. K.; Ding, H. Angew. Chem. Int. Ed. 2001, 40, 4491-4493.

(3) Shen, Y.; Friestad, G. K. J. Org. Chem. 2002, 67, 6236-6239.

(4) (a) Friestad, G. K.; Qin, J. J. Am. Chem. Soc. 2000, 122, 8329-8330. (b) Friestad, G. K.; Draghici, C.; Soukri, M.; Qin, J. J. Org. Chem. 2005, 70, 6330-6338.

(5) Ding, H.; Friestad, G. K. Synthesis 2004, 2216-2221.

(6) (a) Mulzer, J.; Bohm, I.; Bats, J.-W. Tetrahedron Lett. 1998, 39, 9643-9646. (b) Krafft, M. E.; Schmidt, P. Synth. Commun. 2002, 32, 2723-2732.

(7) (a) Harms, A. E.; Stille, J. R.; Taylor, S. K. Organometallics 1994, 13, 1456-1464. (b) Murphy, P. J.; Williams, H. L.; Hibbs, D. E.; Hursthouse, M. B.; Abdul Malik, K. M. Tetrahedron 1996, $52,8315-8332$.

(8) (a) Wang, X.; Li, J.; Zhang, Y. Synth. Commun. 2003, 33, 3575-3581. (b) Katritzky, A. R.; Koeditz, J.; Lang, H. Inorg. Chim. Acta 1994, 220, 67-72. (c) Cook, G. R.; Maity, B. C.; Kargbo, R. Org. Lett. 2004, 6, 1741-1743.

(9) Basile, T.; Bocoum, A.; Savoia, D.; Umani-Ronchi, A. J. Org. Chem. 1994, 59, 7766-7773.

(10) Friestad, G. K.; Qin, J. J. Am. Chem. Soc. 2001, 123, 9922-9923.

(11) (a) Maguire, R. J.; Thomas, E. J. J. Chem. Soc. Perkin Trans. 1 1995, 2477-2485. (b) O’Malley, S. J.; Leighton, J. L. Angew. Chem. Int. Ed. 2001, 40, 2915-2917. 


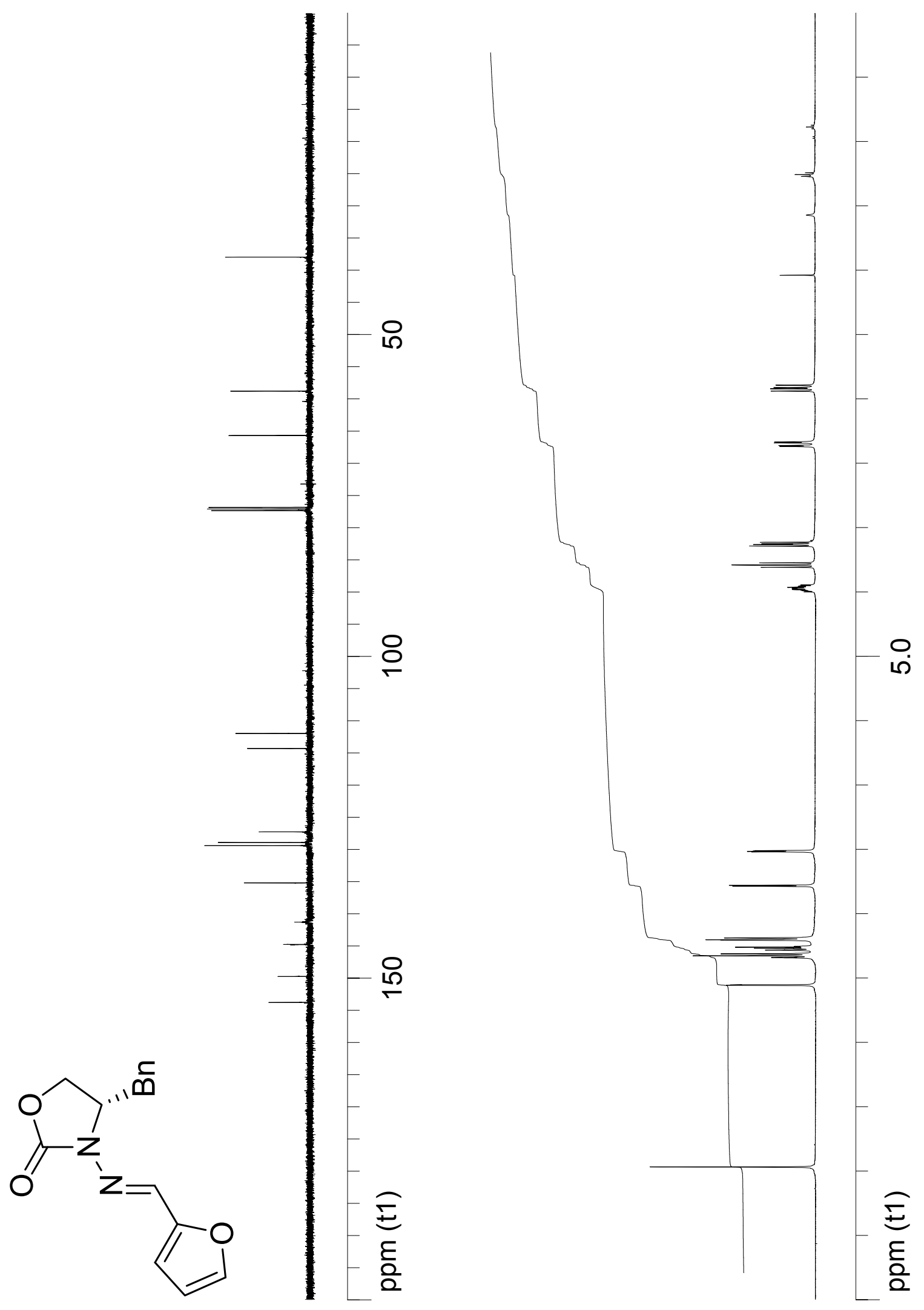

S17 (Compound 1e) 


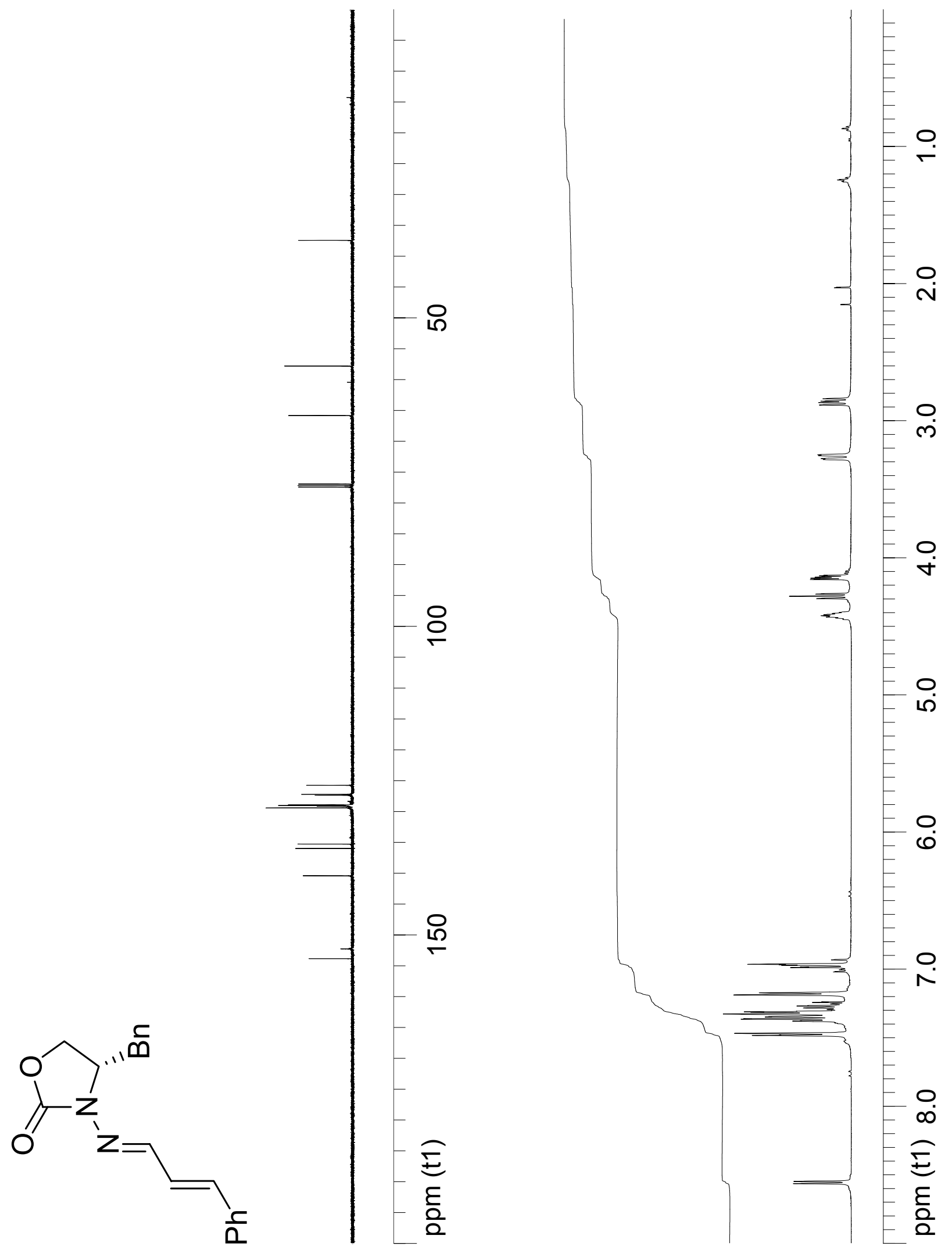



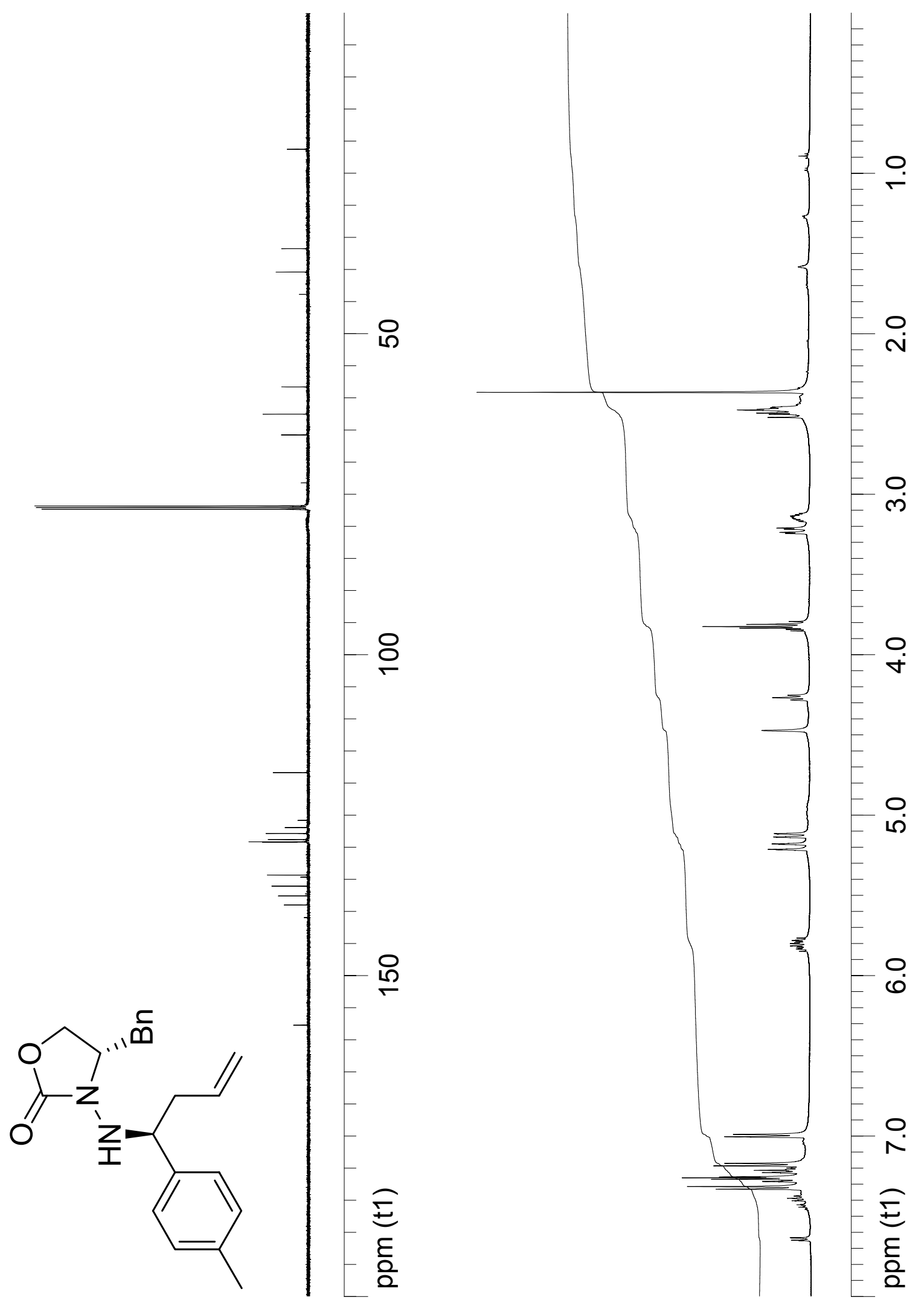


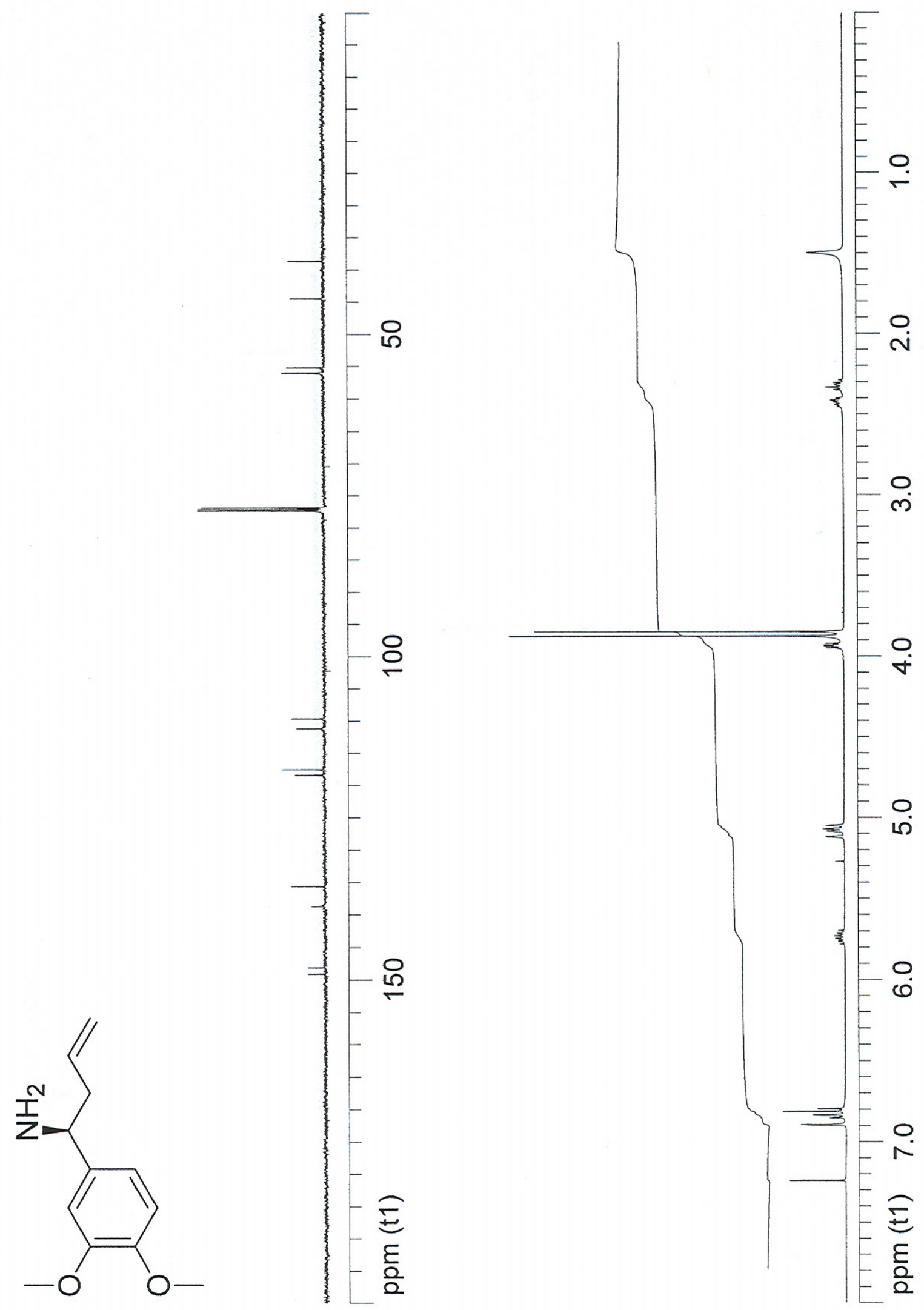




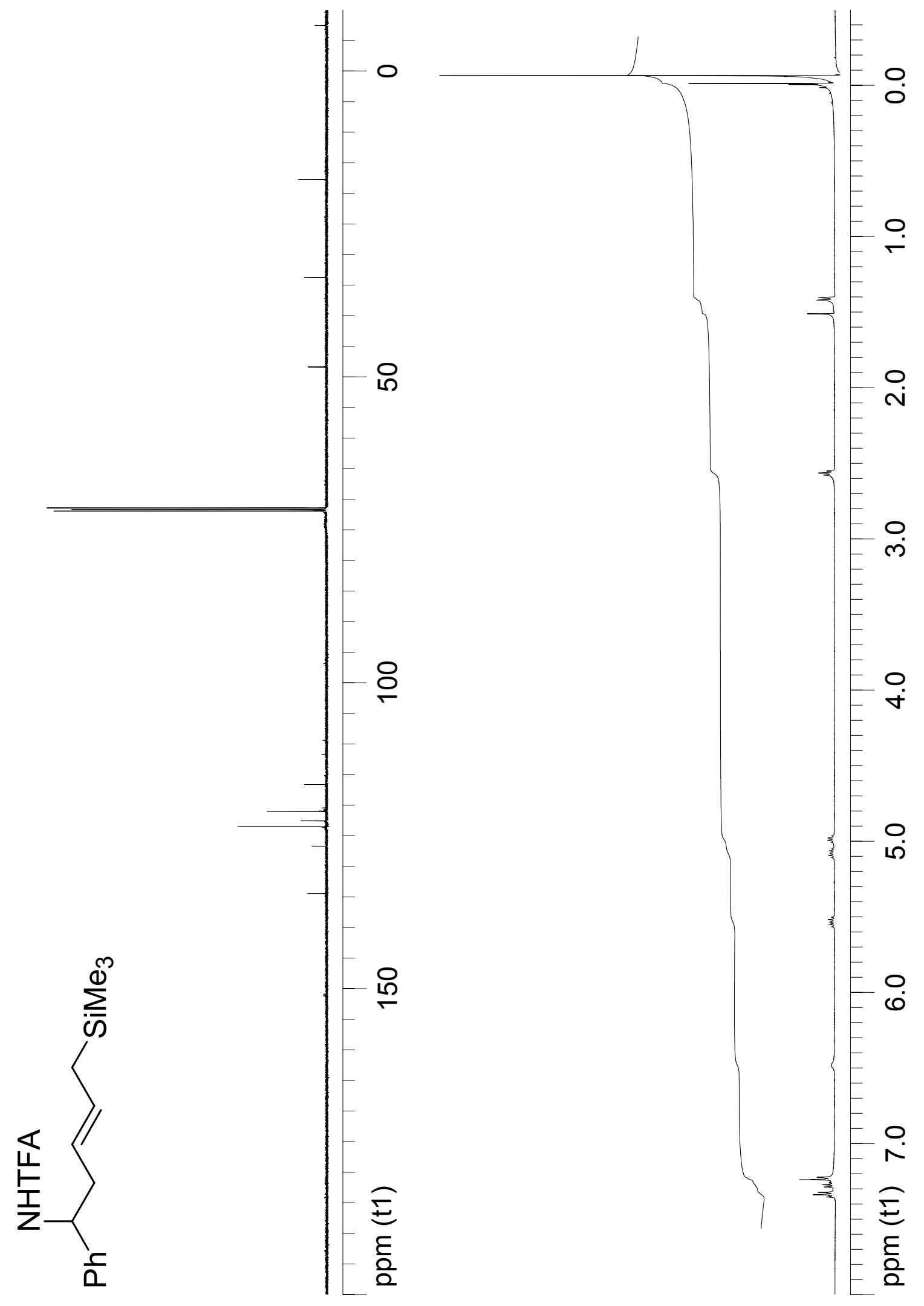




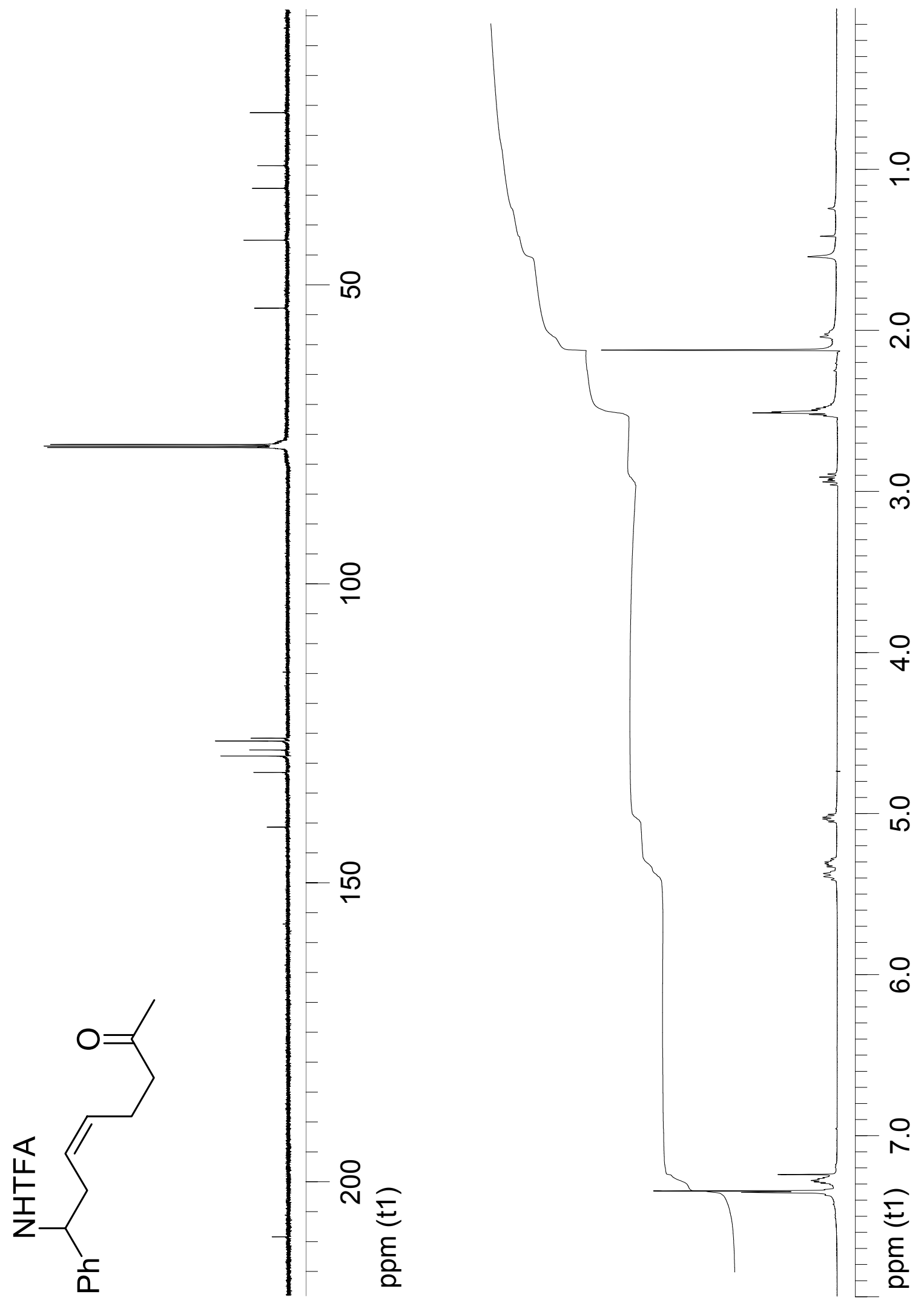

S22 (Compound (Z)-14) 

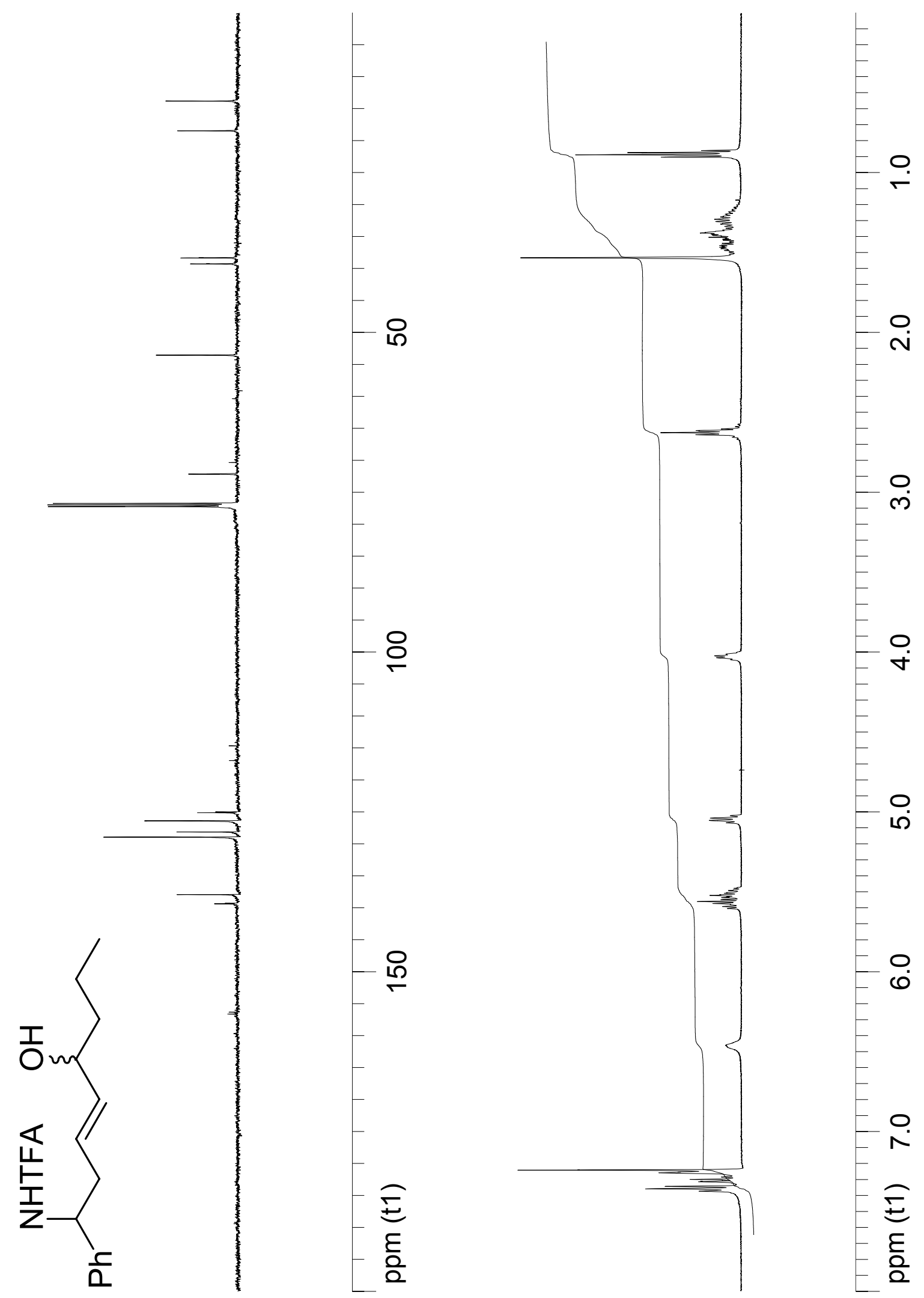

S23 (Compound 15) 


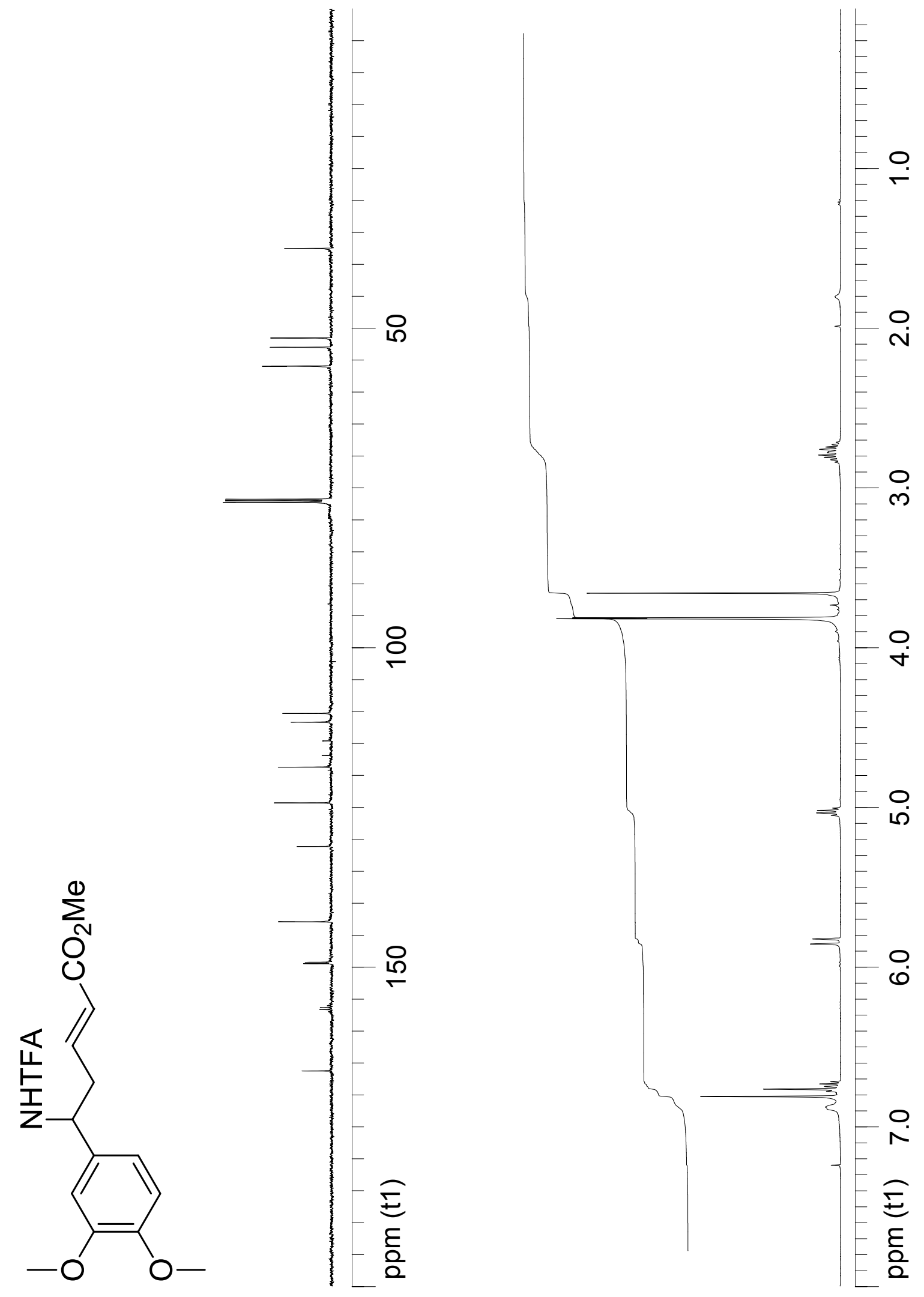

S24 (Compound (E)-17) 


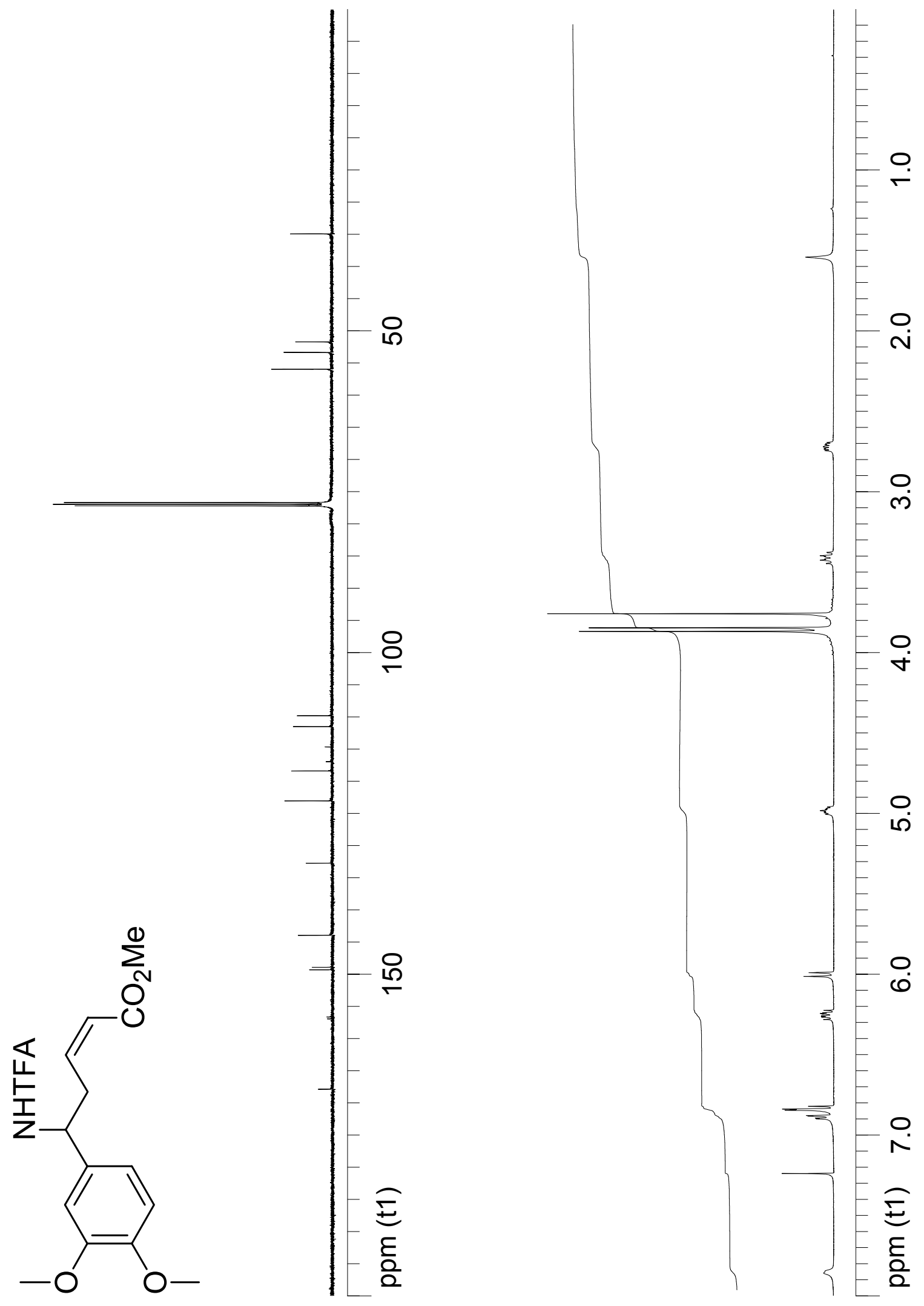



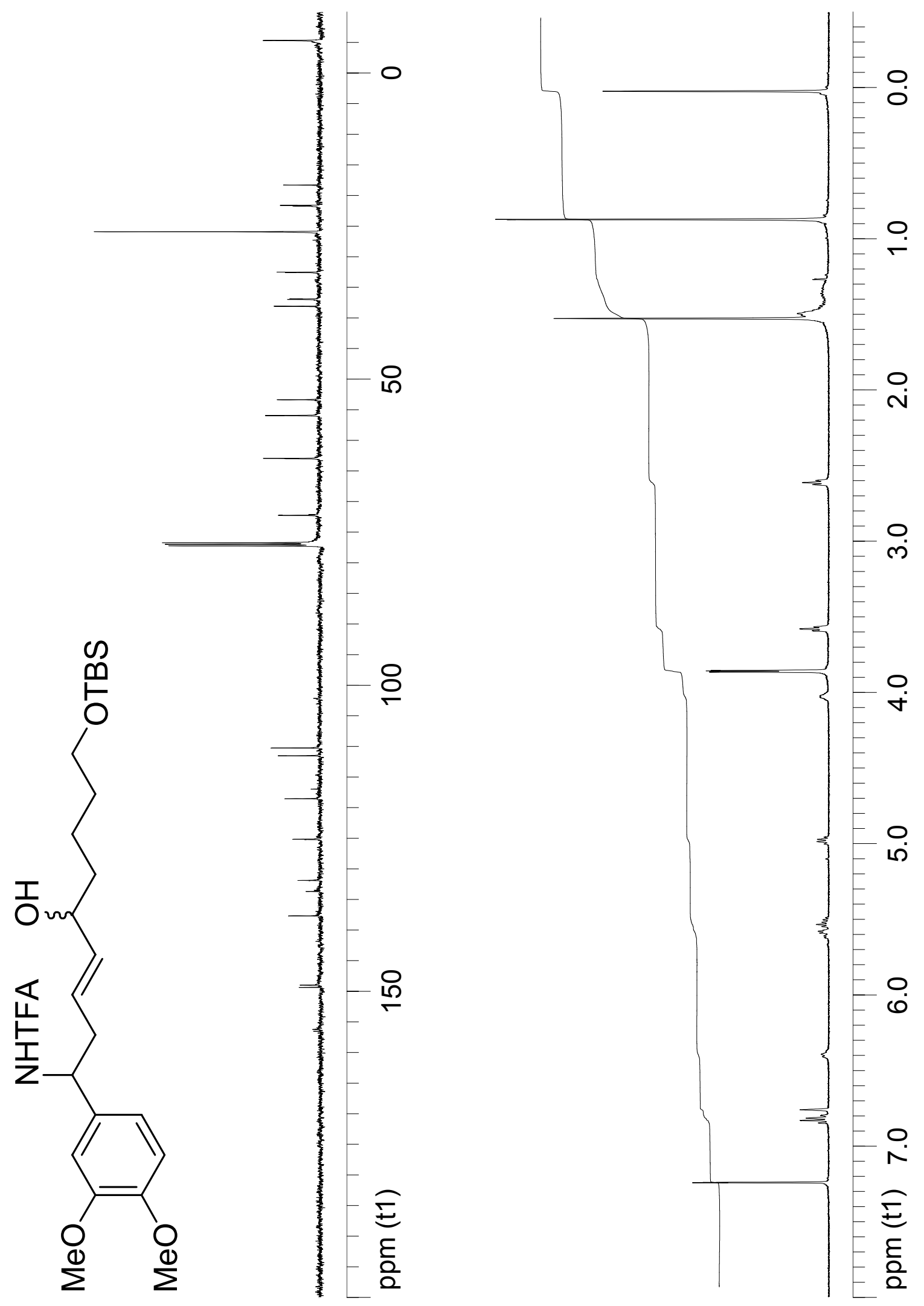


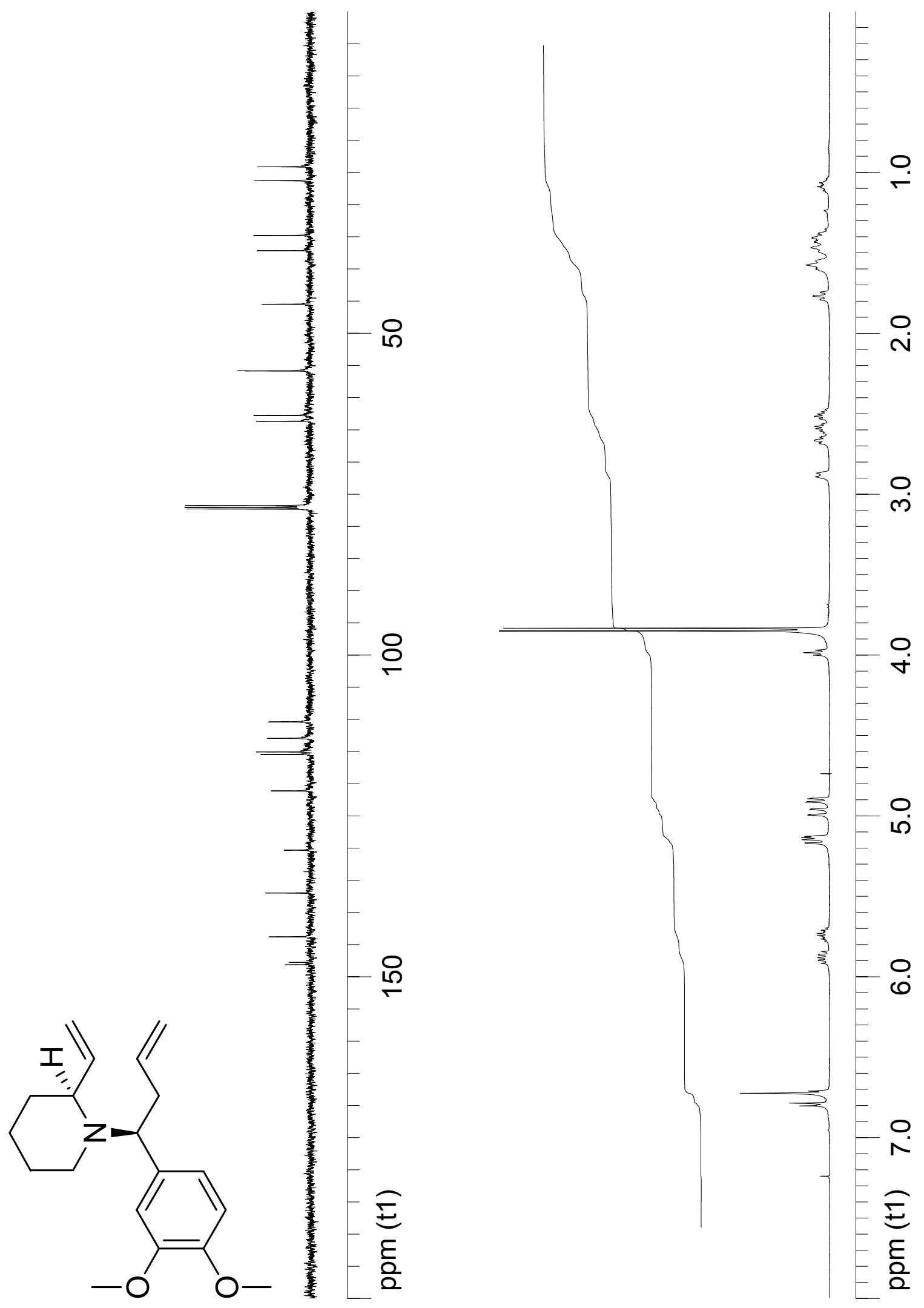




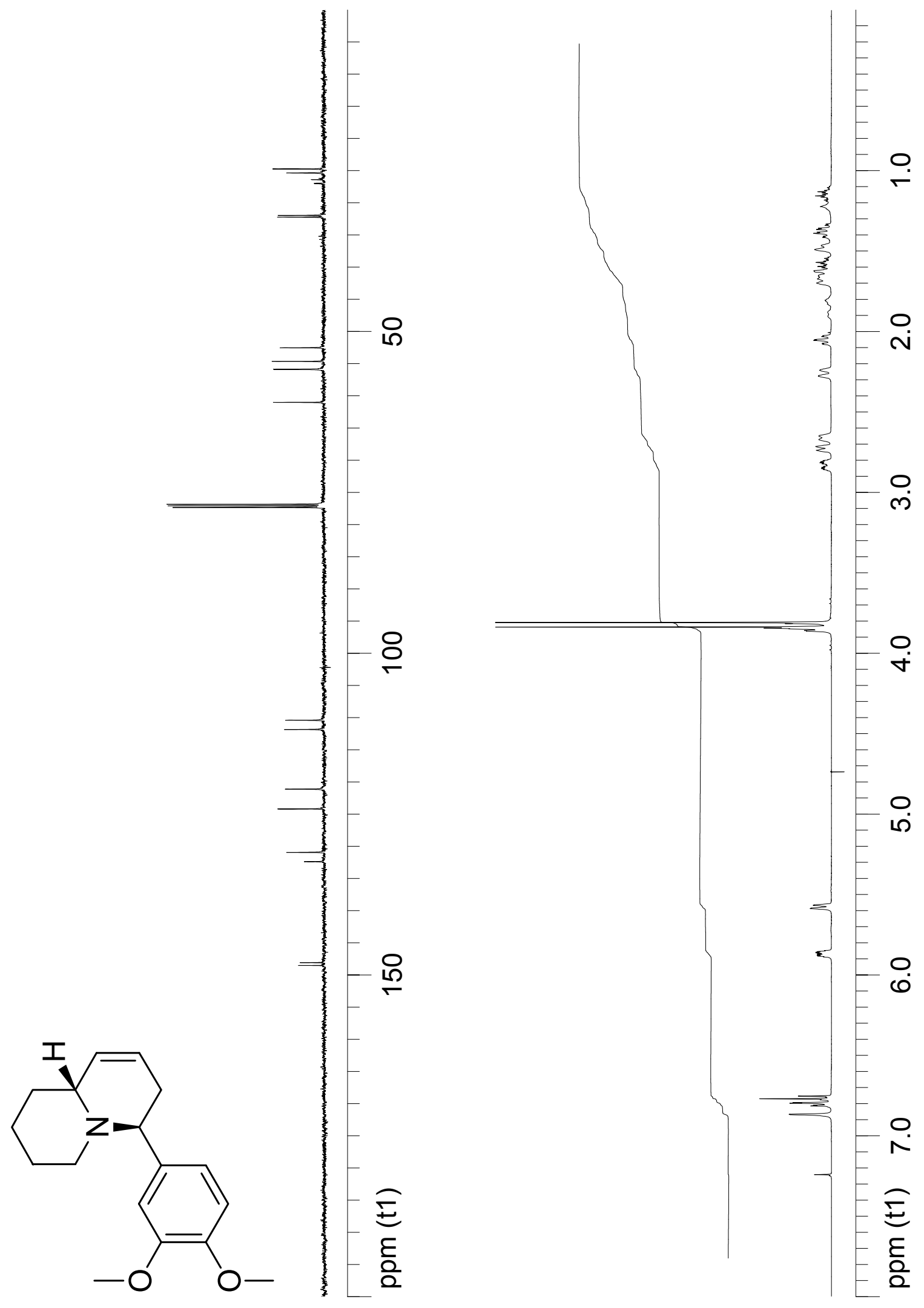

S28 (Compound (9aR)-26) 


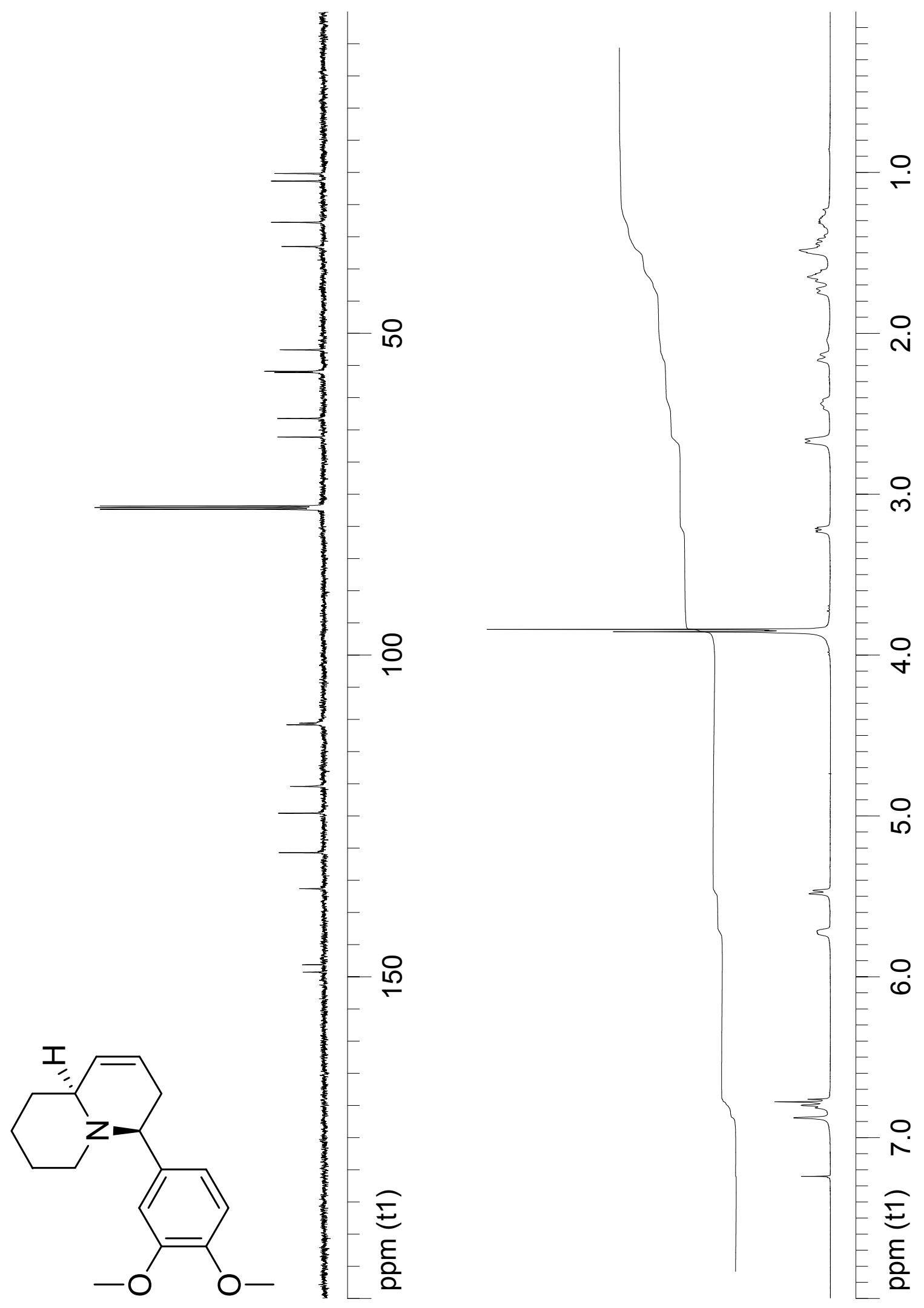

S29 (Compound (9aS)-26) 